\title{
Hadronic uncertainties in the elastic scattering of supersymmetric dark matter
}

\author{
John Ellis, ${ }^{1, *}$ Keith A. Olive, ${ }^{2, \dagger}$ and Christopher Savage ${ }^{2, \dagger}$ \\ ${ }^{1}$ TH Division, Physics Department, CERN, 1211 Geneva 23, Switzerland \\ ${ }^{2}$ William I. Fine Theoretical Physics Institute, School of Physics and Astronomy, University of Minnesota, \\ Minneapolis, Minnesota 55455, USA
}

(Received 28 January 2008; published 27 March 2008)

\begin{abstract}
We review the uncertainties in the spin-independent and spin-dependent elastic scattering cross sections of supersymmetric dark matter particles on protons and neutrons. We propagate the uncertainties in quark masses and hadronic matrix elements that are related to the $\pi$-nucleon $\sigma$ term and the spin content of the nucleon. By far the largest single uncertainty is that in spin-independent scattering induced by our ignorance of the $\langle N|\bar{q} q| N\rangle$ matrix elements linked to the $\pi$-nucleon $\sigma$ term, which affects the ratio of cross sections on proton and neutron targets as well as their absolute values. This uncertainty is already impacting the interpretations of experimental searches for cold dark matter. We plead for an experimental campaign to determine better the $\pi$-nucleon $\sigma$ term. Uncertainties in the spin content of the proton affect significantly, but less strongly, the calculation of rates used in indirect searches.
\end{abstract}

DOI: 10.1103/PhysRevD.77.065026

PACS numbers: 12.60.Jv, 95.35.+d

\section{INTRODUCTION}

The most convincing way to confirm the existence and nature of dark matter particles would be to observe directly their scattering on nuclei in low-background underground experiments. The sensitivities of these experiments are currently improving rapidly and beginning to cut into the parameter space of plausible supersymmetric scenarios [1]. In order to evaluate accurately the impacts of these experiments, it is important to understand and minimize the hadronic uncertainties in the elastic scattering matrix elements for any given supersymmetric model. The rates for elastic scattering also control the rates for the capture of dark matter particles by celestial bodies such the Sun or Earth. There are good prospects for increasing significantly the sensitivities of experiments looking indirectly for astrophysical dark matter via the products of their annihilations in such bodies, adding to the motivations for understanding and reducing their uncertainties.

Beyond the interpretation of upper limits on dark matter scattering may lie the interpretation of any eventual detection of a signal and the task of identifying the nature of the dark matter particle. In principle, there are four observables that could contribute to such an analysis, namely, the spin-independent and spin-dependent cross sections on protons and neutrons, respectively. Part of the strategy for identifying the nature of any detected dark matter signal would be the comparison of the measured rates for scattering on different targets, with the comparison of spinindependent and spin-dependent scattering rates playing a particularly important role as a diagnostic tool $[2,3]$. As we see later, currently there are considerable uncertainties also in such comparisons, related principally to uncertainties in

\footnotetext{
*John.Ellis@cern.ch

†olive@physics.umn.edu

†cmsavage@physics.umn.edu
}

the hadronic matrix elements of higher-dimensional effective interactions.

In this paper, we consider only hadronic uncertainties in the elastic scattering rates. There are also potentially important uncertainties related to the supersymmetric model itself, namely, how accurately one can estimate the coefficient of a given higher-dimensional effective interaction in a given model, and also in the astrophysical density of dark matter particles. Reducing the model uncertainty would require, e.g., a complete calculation of radiative corrections to the effective scattering operator, which lies beyond the scope of this work. As for the local density of cold dark matter, it is usually taken to be $0.3 \mathrm{GeV} / \mathrm{cm}^{3}$, but lower values have occasionally been advocated.

The hadronic uncertainties we consider are listed in Table I. They include those in the quark masses, expressed as $m_{\mathrm{d}, \mathrm{c}, \mathrm{b}, \mathrm{t}}$ and the ratios $m_{\mathrm{u}} / m_{\mathrm{d}}$ and $m_{\mathrm{s}} / m_{\mathrm{d}}$, those in the matrix elements $\langle N|\bar{q} q| N\rangle$, which are related to the change in the nucleon mass due to nonzero quark masses, denoted

TABLE I. Hadronic parameters used to determine neutralinonucleon scattering cross sections, with estimates of their experimental uncertainties.

\begin{tabular}{lcc}
\hline \hline$m_{\mathrm{u}} / m_{\mathrm{d}}$ & $0.553 \pm 0.043$ & {$[4]$} \\
$m_{\mathrm{d}}$ & $5 \pm 2 \mathrm{MeV}$ & {$[5]$} \\
$m_{\mathrm{s}} / m_{\mathrm{d}}$ & $18.9 \pm 0.8$ & {$[4]$} \\
$m_{\mathrm{c}}$ & $1.25 \pm 0.09 \mathrm{GeV}$ & {$[5]$} \\
$m_{\mathrm{b}}$ & $4.20 \pm 0.07 \mathrm{GeV}$ & {$[5]$} \\
$m_{\mathrm{t}}$ & $171.4 \pm 2.1 \mathrm{GeV}$ & {$[6]$} \\
$\sigma_{0}$ & $36 \pm 7 \mathrm{MeV}$ & {$[7]$} \\
$\Sigma_{\pi N}$ & $64 \pm 8 \mathrm{MeV}$ & {$[8,9]$} \\
$a_{3}^{(\mathrm{p})}$ & $1.2695 \pm 0.0029$ & {$[5]$} \\
$a_{8}^{(\mathrm{p})}$ & $0.585 \pm 0.025$ & {$[10,11]$} \\
$\Delta_{\mathrm{s}}^{(\mathrm{p})}$ & $-0.09 \pm 0.03$ & {$[12]$} \\
\hline \hline
\end{tabular}


by $\sigma_{0}$, and therefore to the $\pi$-nucleon $\sigma$ term, $\Sigma_{\pi N}$ as discussed later, and the axial-current matrix elements $\left\langle N\left|\bar{q} \gamma_{\mu} \gamma_{5} q\right| N\right\rangle$, which are related to the quantities $\Delta_{\mathrm{s}}^{(\mathrm{p})}$, $a_{3}^{(\mathrm{p})}$, and $a_{8}^{(\mathrm{p})}$, as also discussed later. We find that the uncertainties in the elastic scattering cross section induced by the uncertainties in the quark masses, apart from the top quark, are negligible. However, cross section uncertainties induced by the uncertainties in the matrix elements $\langle N|\bar{q} q| N\rangle$ and $\left\langle N\left|\bar{q} \gamma_{\mu} \gamma_{5} q\right| N\right\rangle$ are very important, as we discuss below. In particular, the uncertainties induced by our ignorance of $\Sigma_{\pi N}$ are particularly important.

For illustration, we work within the minimal supersymmetric extension of the standard model (MSSM) with conserved $R$ parity, and assume that the astrophysical cold dark matter is provided by the lightest neutralino $\chi$ [13]. We further assume a constrained MSSM (CMSSM) framework, in which the soft supersymmetry-breaking parameters $m_{1 / 2}, m_{0}$ and $A_{0}$ are assumed to be universal at the GUT input scale, and restrict our attention to scenarios with the Higgs mixing parameter $\mu>0$ and specific values of the ratio of supersymmetric Higgs v.e.v.s $\tan \beta$ [14]. We illustrate our observations by studies of some specific CMSSM benchmark scenarios [15], and also by surveys along strips in the $\left(m_{1 / 2}, m_{0}\right)$ plane for $\tan \beta=10$, 50 along which $\tilde{\tau}-\chi$ coannihilation maintains the relic neutralino density within the range favored by WMAP and other experiments [16].

We find that the spin-independent cross section may vary by almost an order of magnitude for $48 \mathrm{MeV}$ $<\Sigma_{\pi N}<80 \mathrm{MeV}$, the $\pm 2-\sigma$ range according to the uncertainties in Table I. This uncertainty is already impacting the interpretations of experimental searches for cold dark matter. Propagating the $\pm 2-\sigma$ uncertainties in $\Delta_{\mathrm{s}}^{(\mathrm{p})}$, the next most important parameter, we find a variation by a factor $\sim 2$ in the spin-dependent cross section. Since the spin-independent cross section may now be on the verge of detectability in certain models, and the uncertainty in the cross section is far greater, we appeal for a greater, dedicated effort to reduce the experimental uncertainty in the $\pi$-nucleon $\sigma$ term $\Sigma_{\pi N}$. This quantity is not just an object of curiosity for those interested in the structure of the nucleon and nonperturbative strong-interaction effects: it may also be key to understanding new physics beyond the standard model.

\section{SUPERSYMMETRIC FRAMEWORK}

We briefly review in this section the theoretical framework we use in the context of the MSSM; for more comprehensive reviews, see, e.g., $[17,18]$. The neutralino LSP is the lowest-mass eigenstate combination of the bino $\tilde{B}$, wino $\tilde{W}$, and Higgsinos $\tilde{H}_{1,2}$, whose mass matrix $N$ is diagonalized by a matrix $Z$ : $\operatorname{diag}\left(m_{\chi_{1, \ldots 4}}\right)=Z^{*} N Z^{-1}$. The composition of the lightest neutralino may be written as

$$
\chi=Z_{\chi 1} \tilde{B}+Z_{\chi^{2}} \tilde{W}+Z_{\chi^{3}} \tilde{H}_{1}+Z_{\chi^{4}} \tilde{H}_{2} \text {. }
$$

As already mentioned, we work here in the context of the CMSSM and assume universality at the supersymmetric GUT scale for the gaugino masses, $m_{1 / 2}$, as well as for the soft scalar masses, $m_{0}$, and trilinear terms, $A_{0}$. Our treatment of the sfermion mass matrices $M$ follows [19,20]. The sfermion mass-squared matrix is diagonalized by a matrix $\eta: \operatorname{diag}\left(m_{1}^{2}, m_{2}^{2}\right) \equiv \eta M^{2} \eta^{-1}$, which can be parametrized for each flavour $f$ by an angle $\theta_{f}$. We ignore here all possible $C P$-violating phases. The diagonalization matrix can be written as

$$
\left(\begin{array}{cc}
\cos \theta_{f} & \sin \theta_{f} e^{i \gamma_{f}} \\
-\sin \theta_{f} e^{-i \gamma_{f}} & \cos \theta_{f}
\end{array}\right) \equiv\left(\begin{array}{ll}
\eta_{11} & \eta_{12} \\
\eta_{21} & \eta_{22}
\end{array}\right)
$$

The magnitudes of $\mu$ and the pseudoscalar Higgs mass $m_{A}$ are calculated from the electroweak vacuum conditions using $m_{\mathrm{t}}=171.4 \mathrm{GeV}$, except as noted in Sec. IV.

The only four-fermi Lagrangian contributions for describing elastic $\chi$-nucleon scattering obtained from the MSSM Lagrangian which are not velocity dependent, and hence relevant for relic dark matter scattering, are [19]:

$$
\mathcal{L}=\alpha_{2 i} \bar{\chi} \gamma^{\mu} \gamma^{5} \chi \bar{q}_{i} \gamma_{\mu} \gamma^{5} q_{i}+\alpha_{3 i} \bar{\chi} \chi \bar{q}_{i} q_{i} .
$$

This Lagrangian is to be summed over the quark generations, and the subscript $i$ labels up-type quarks $(i=1)$ and down-type quarks $(i=2)$. The coefficients are given by:

$$
\begin{aligned}
\alpha_{2 i}= & \frac{1}{4\left(m_{1 i}^{2}-m_{\chi}^{2}\right)}\left[\left|X_{i}\right|^{2}+\left|Y_{i}\right|^{2}\right]+\frac{1}{4\left(m_{2 i}^{2}-m_{\chi}^{2}\right)} \\
& \times\left[\left|W_{i}\right|^{2}+\left|V_{i}\right|^{2}\right]-\frac{g^{2}}{4 m_{Z}^{2} \cos ^{2} \theta_{W}} \\
& \times\left[\left|Z_{\chi_{3}}\right|^{2}-\left|Z_{\chi_{4}}\right|^{2}\right] \frac{T_{3 i}}{2}
\end{aligned}
$$

and

$$
\begin{aligned}
\alpha_{3 i}= & -\frac{1}{2\left(m_{1 i}^{2}-m_{\chi}^{2}\right)} \operatorname{Re}\left[\left(X_{i}\right)\left(Y_{i}\right)^{*}\right] \\
& -\frac{1}{2\left(m_{2 i}^{2}-m_{\chi}^{2}\right)} \operatorname{Re}\left[\left(W_{i}\right)\left(V_{i}\right)^{*}\right] \\
& -\frac{g m_{q_{i}}}{4 m_{W} B_{i}}\left\{\left(\frac{D_{i}^{2}}{m_{H_{2}}^{2}}+\frac{C_{i}^{2}}{m_{H_{1}}^{2}}\right) \operatorname{Re}\left[\delta_{2 i}\left(g Z_{\chi 2}-g^{\prime} Z_{\chi 1}\right)\right]\right. \\
& \left.+D_{i} C_{i}\left(\frac{1}{m_{H_{2}}^{2}}-\frac{1}{m_{H_{1}}^{2}}\right) \operatorname{Re}\left[\delta_{1 i}\left(g Z_{\chi^{2}}-g^{\prime} Z_{\chi 1}\right)\right]\right\},
\end{aligned}
$$

where 


$$
\begin{aligned}
X_{i} & \equiv \eta_{11}^{*} \frac{g m_{q_{i}} Z_{\chi 5-i}^{*}}{2 m_{W} B_{i}}-\eta_{12}^{*} e_{i} g^{\prime} Z_{\chi 1}^{*} \\
Y_{i} & \equiv \eta_{11}^{*}\left(\frac{y_{i}}{2} g^{\prime} Z_{\chi 1}+g T_{3 i} Z_{\chi 2}\right)+\eta_{12}^{*} \frac{g m_{q_{i}} Z_{\chi 5-i}}{2 m_{W} B_{i}} \\
W_{i} & \equiv \eta_{21}^{*} \frac{g m_{q_{i}} Z_{\chi 5-i}^{*}}{2 m_{W} B_{i}}-\eta_{22}^{*} e_{i} g^{\prime} Z_{\chi 1}^{*} \\
V_{i} & \equiv \eta_{21}^{*}\left(\frac{y_{i}}{2} g^{\prime} Z_{\chi 1}+g T_{3 i} Z_{\chi 2}\right)+\eta_{22}^{*} \frac{g m_{q_{i}} Z_{\chi 5-i}}{2 m_{W} B_{i}},
\end{aligned}
$$

where $y_{i}, T_{3 i}$ denote hypercharge and isospin, and

$$
\begin{gathered}
\delta_{1 i}=Z_{\chi^{3}}\left(Z_{\chi^{4}}\right), \quad \delta_{2 i}=Z_{\chi^{4}}\left(-Z_{\chi^{3}}\right), \\
B_{i}=\sin \beta(\cos \beta), \quad C_{i}=\sin \alpha(\cos \alpha), \\
D_{i}=\cos \alpha(-\sin \alpha)
\end{gathered}
$$

for up (down) type quarks. We denote by $m_{H_{2}}<m_{H_{1}}$ the masses of the two neutral scalar Higgs bosons, and $\alpha$ denotes the neutral Higgs boson mixing angle.

\section{HADRONIC MATRIX ELEMENTS}

The elastic cross section for neutralino scattering off a nucleus can be decomposed into a scalar (spinindependent) part obtained from the $\alpha_{3 i}$ term in Eq. (3), and a spin-dependent part obtained from the $\alpha_{2 i}$ term. Each of these can be written in terms of the cross sections for elastic scattering for scattering off individual nucleons, as we now review and reevaluate.

\section{A. Spin-independent term}

The scalar, or spin-independent (SI), part of the cross section can be written as ${ }^{1}$

$$
\sigma_{\mathrm{SI}}=\frac{4 m_{r}^{2}}{\pi}\left[Z f_{p}+(A-Z) f_{n}\right]^{2},
$$

where $m_{r}$ is the $\chi$-nuclear reduced mass and

$$
\frac{f_{N}}{m_{N}}=\sum_{q=\mathrm{u}, \mathrm{d}, \mathrm{s}} f_{T_{q}}^{(N)} \frac{\alpha_{3 q}}{m_{q}}+\frac{2}{27} f_{T G}^{(N)} \sum_{q=\mathrm{c}, \mathrm{b}, \mathrm{t}} \frac{\alpha_{3 q}}{m_{q}}
$$

for $N=$ p or n. The parameters $f_{T_{q}}^{(N)}$ are defined by

$$
m_{N} f_{T_{q}}^{(N)} \equiv\left\langle N\left|m_{q} \bar{q} q\right| N\right\rangle \equiv m_{q} B_{q}^{(N)}
$$

where $[22,23]$

$$
f_{T G}^{(N)}=1-\sum_{q=\mathrm{u}, \mathrm{d}, \mathrm{s}} f_{T_{q}}^{(N)} .
$$

We take the ratios of the light quark masses from [4]:

\footnotetext{
${ }^{1}$ This expression is valid in the zero-momentum-transfer limit. For nonzero momentum exchange, the expression must include a form factor due to the finite size of the nucleus. See, for example, Ref. [21].
}

$$
\frac{m_{\mathrm{u}}}{m_{\mathrm{d}}}=0.553 \pm 0.043, \quad \frac{m_{\mathrm{s}}}{m_{\mathrm{d}}}=18.9 \pm 0.8 .
$$

We take the other quark masses from [5], except for the top mass, which is taken from the combined CDF and D0 result [6]. These masses, as well as other experimental quantities that will arise in the calculation of the hadronic matrix elements, appear in Table I.

Following [24], we introduce the quantity:

$$
z \equiv \frac{B_{\mathrm{u}}^{(\mathrm{p})}-B_{\mathrm{s}}^{(\mathrm{p})}}{B_{\mathrm{d}}^{(\mathrm{p})}-B_{\mathrm{s}}^{(\mathrm{p})}}=1.49,
$$

which has an experimental error that is negligible compared with others discussed below, and the strange scalar density

$$
y \equiv \frac{2 B_{\mathrm{s}}^{(N)}}{B_{\mathrm{u}}^{(N)}+B_{\mathrm{d}}^{(N)}} .
$$

In terms of these, one may write

$$
\frac{B_{\mathrm{d}}^{(\mathrm{p})}}{B_{\mathrm{u}}^{(\mathrm{p})}}=\frac{2+((z-1) \times y)}{2 \times z-((z-1) \times y)} .
$$

Proton and neutron scalar matrix elements are related by an interchange of $B_{\mathrm{u}}$ and $B_{\mathrm{d}}$, i.e.,

$$
B_{\mathrm{u}}^{(\mathrm{n})}=B_{\mathrm{d}}^{(\mathrm{p})}, \quad B_{\mathrm{d}}^{(\mathrm{n})}=B_{\mathrm{u}}^{(\mathrm{p})}, \quad \text { and } \quad B_{\mathrm{s}}^{(\mathrm{n})}=B_{\mathrm{s}}^{(\mathrm{p})} .
$$

The $\pi$-nucleon sigma term, $\Sigma_{\pi N}$, may be written as

$$
\Sigma_{\pi N} \equiv \frac{1}{2}\left(m_{\mathrm{u}}+m_{\mathrm{d}}\right) \times\left(B_{\mathrm{u}}^{(N)}+B_{\mathrm{d}}^{(N)}\right)
$$

and the coefficients $f_{T_{q}}$ may be written in the forms:

$$
\begin{gathered}
f_{T_{\mathrm{u}}}=\frac{m_{\mathrm{u}} B_{\mathrm{u}}}{m_{N}}=\frac{2 \Sigma_{\pi N}}{m_{N}\left(1+\frac{m_{\mathrm{d}}}{m_{\mathrm{u}}}\right)\left(1+\frac{B_{\mathrm{d}}}{B_{\mathrm{u}}}\right)}, \\
f_{T_{\mathrm{d}}}=\frac{m_{d} B_{d}}{m_{N}}=\frac{2 \Sigma_{\pi N}}{m_{N}\left(1+\frac{m_{\mathrm{u}}}{m_{\mathrm{d}}}\right)\left(1+\frac{B_{\mathrm{u}}}{B_{\mathrm{d}}}\right)}, \\
f_{T_{\mathrm{s}}}=\frac{m_{\mathrm{s}} B_{\mathrm{s}}}{m_{N}}=\frac{\left(\frac{m_{\mathrm{s}}}{m_{\mathrm{d}}}\right) \Sigma_{\pi N} y}{m_{N}\left(1+\frac{m_{\mathrm{u}}}{m_{\mathrm{d}}}\right)} ;
\end{gathered}
$$

where we have dropped the $(N)$ superscript from $f_{T_{q}}$ and $B_{q}$.

The effect of the uncertainties in the $f_{T_{q}}$ were considered in $[25,26]$ and we were motivated to reconsider [9] the value of $y$ by recent reevaluations of the $\pi$-nucleon sigma term $\Sigma_{\pi N}$, which is related to the strange scalar density in the nucleon by

$$
y=1-\sigma_{0} / \Sigma_{\pi N} .
$$

The value for $\sigma_{0}$ given in Table I is estimated on the basis of octet baryon mass differences to be $\sigma_{0}=36 \pm 7 \mathrm{MeV}$ [7,27-29]. Recent determinations of $\Sigma_{\pi N}$ have found the 
following values at the Cheng-Dashen point $t=+2 m_{\pi}^{2}$ [8]:

$$
\Sigma_{\mathrm{CD}}=(88 \pm 15,71 \pm 9,79 \pm 7,85 \pm 5) \mathrm{MeV} .
$$

These should be corrected by an amount $-\Delta_{R}-\Delta_{\sigma} \simeq$ $-15 \mathrm{MeV}$ to obtain $\Sigma_{\pi N}$. Assuming for definiteness the value $\Sigma_{\mathrm{CD}}=79 \pm 7 \mathrm{MeV}$, one finds

$$
\Sigma_{\pi N}=(64 \pm 8) \mathrm{MeV} \text {. }
$$

This is the range generally considered in this paper, though one could even argue for a larger uncertainty, and we also discuss the implications if $\Sigma_{\pi N}=\sigma_{0}$, i.e., $y=0$.

\section{B. Spin-dependent term}

The spin-dependent (SD) part of the elastic $\chi$-nucleus cross section can be written as ${ }^{2}$

$$
\sigma_{\mathrm{SD}}=\frac{32}{\pi} G_{F}^{2} m_{r}^{2} \Lambda^{2} J(J+1),
$$

where $m_{r}$ is again the reduced neutralino mass, $J$ is the spin of the nucleus,

$$
\Lambda \equiv \frac{1}{J}\left(a_{\mathrm{p}}\left\langle S_{\mathrm{p}}\right\rangle+a_{\mathrm{n}}\left\langle S_{\mathrm{n}}\right\rangle\right)
$$

and

$$
a_{\mathrm{p}}=\sum_{q} \frac{\alpha_{2 q}}{\sqrt{2} G_{f}} \Delta_{q}^{(\mathrm{p})}, \quad a_{\mathrm{n}}=\sum_{i} \frac{\alpha_{2 q}}{\sqrt{2} G_{f}} \Delta_{q}^{(\mathrm{n})} .
$$

The factors $\Delta_{q}^{(N)}$ parametrize the quark spin content of the nucleon and are only significant for the light $(\mathrm{u}, \mathrm{d}, \mathrm{s})$ quarks. A combination of experimental and theoretical results tightly constrain the linear combinations [5]

$$
a_{3}^{(\mathrm{p})} \equiv \Delta_{\mathrm{u}}^{(\mathrm{p})}-\Delta_{\mathrm{d}}^{(\mathrm{p})}=1.2695 \pm 0.0029
$$

and $[10,11]$

$$
a_{8}^{(\mathrm{p})} \equiv \Delta_{\mathrm{u}}^{(\mathrm{p})}+\Delta_{\mathrm{d}}^{(\mathrm{p})}-2 \Delta_{\mathrm{s}}^{(\mathrm{p})}=0.585 \pm 0.025 .
$$

However, the individual $\Delta_{q}^{(N)}$ are relatively poorly constrained; using the recent COMPASS result [12],

$$
\Delta_{\mathrm{s}}^{(\mathrm{p})}=-0.09 \pm 0.01(\text { stat }) \pm 0.02(\text { syst }) \approx-0.09 \pm 0.03 \text {, }
$$

where we have conservatively combined the statistical and systematic uncertainties, we may express $\Delta_{\mathrm{u}, \mathrm{d}}^{(N)}$ as follows in terms of known quantities:

$$
\Delta_{\mathrm{u}}^{(p)}=\frac{1}{2}\left(a_{8}^{(\mathrm{p})}+a_{3}^{(\mathrm{p})}\right)+\Delta_{s}^{(\mathrm{p})}=0.84 \pm 0.03,
$$

\footnotetext{
${ }^{2}$ As with the SI cross section, this expression applies in the zero momentum transfer limit and requires an additional form factor for finite momentum transfer. This form factor may have a small but nonzero dependence on $a_{\mathrm{p}}$ and $a_{\mathrm{n}}$.
}

$$
\Delta_{\mathrm{d}}^{(\mathrm{p})}=\frac{1}{2}\left(a_{8}^{(\mathrm{p})}-a_{3}^{(\mathrm{p})}\right)+\Delta_{\mathrm{s}}^{(\mathrm{p})}=-0.43 \pm 0.03 .
$$

The above two uncertainties and that of $\Delta_{\mathrm{s}}^{(\mathrm{p})}$, however, are correlated and we shall instead use the independent quantities $a_{3}^{(\mathrm{p})}$ and $a_{8}^{(\mathrm{p})}$ when appropriate. These values differ by approximately $2 \sigma$ from those used in [25] and we will explore the impact of this change on the magnitude and ratios of the spin-dependent cross sections. The proton and neutron scalar matrix elements are related by an interchange of $\Delta_{\mathrm{u}}$ and $\Delta_{\mathrm{d}}$, or

$$
\Delta_{\mathrm{u}}^{(\mathrm{n})}=\Delta_{\mathrm{d}}^{(\mathrm{p})}, \quad \Delta_{\mathrm{d}}^{(\mathrm{n})}=\Delta_{\mathrm{u}}^{(\mathrm{p})}, \quad \text { and } \quad \Delta_{\mathrm{s}}^{(\mathrm{n})}=\Delta_{\mathrm{s}}^{(\mathrm{p})} .
$$

\section{SCATTERING CROSS SECTIONS, RATIOS AND UNCERTAINTIES}

Direct and some indirect dark matter detection techniques involve the scattering of a weakly interactive massive particle (WIMP) off a nucleus. Direct detection experiments, such as CDMS [30,31], XENON10 [32,33], ZEPLIN-II [34,35], and KIMS [36] aim to detect dark matter via scattering of relic neutralinos off nuclei inside the detectors [37]. Indirect detection experiments such as Super-Kamiokande [38] and AMANDA/IceCube [39], on the other hand, search for high-energy neutrinos produced in WIMP annihilations at the center of the Sun [40] or Earth [41]. The annihilations are the result of WIMPs accumulating at the centers of these massive bodies due to galactic WIMPs scattering off nuclei in these bodies and losing enough energy to become gravitationally bound. Subsequent scatters then cause the WIMPs to fall to the cores, where the local density is enhanced. Other indirect detection methods search for WIMPs that annihilate in the galactic halo or near the galactic center, where they produce neutrinos, positrons, or antiprotons that may be seen in detectors on the Earth [42,43]. However, such annihilations do not involve scattering off nuclei, and will not be discussed here.

The rate of such scattering events and the relative sensitivities of different experiments are dependent upon the four $\chi$-nucleon scattering cross sections (spin-independent (SI) and spin-dependent (SD) cross sections for each of the proton and neutron). The interpretation of any positive signal in such an experiment requires an understanding of the precision that such a signal can be correlated with underlying parameters within a given theoretical framework (e.g., $m_{0}, m_{1 / 2}$, and $\tan \beta$ in the CMSSM).

Direct and indirect detection signals are inherently proportional to $\rho_{0} \sigma_{\chi N}$, rather than $\rho_{0}$ or $\sigma_{\chi^{N}}$ independently, where $\rho_{0}$ is the local density of dark matter (assumed here to be dominated by relic neutralinos) and $\sigma_{\chi^{N}}$ is any of the four $\chi$-nucleon cross sections. Since the local dark matter density has not been directly measured, it is typically inferred from galactic dynamics and $N$-body simulations. By convention, experimental results are often presented for 
a neutralino density of $0.3 \mathrm{GeV} / \mathrm{cm}^{3}$, with the implicit understanding that the following significant uncertainties exist in this value. In the case of a smooth distribution of galactic dark matter, $\rho_{0}$ is estimated to be $0.2-0.4 \mathrm{GeV} / \mathrm{cm}^{3}[44,45]$ for a spherical halo, but it may be somewhat higher, up to $0.7 \mathrm{GeV} / \mathrm{cm}^{3}$ [46,47], for an elliptical halo; see Ref. [45] for a discussion of the difficulties in determining this value. Models of the galaxy based upon hierarchical formation [48], which do not assume a strictly smooth distribution of dark matter as do the above estimates, suggest that the local density may be as low as $0.04 \mathrm{GeV} / \mathrm{cm}^{3}$, but that $0.2 \mathrm{GeV} / \mathrm{cm}^{3}$ is a more reasonable lower limit. With this hierarchical formation, the halo may contain substructure such as clumps [49,50] or tidal streams from galaxy mergers [51] that can increase the dark matter density. An analysis of the Sagittarius dwarf galaxy, whose leading tidal tail passes through the galactic disk somewhere near our location, estimates it may contribute an additional $0.001-0.07 \mathrm{GeV} / \mathrm{cm}^{3}$ to the local dark matter density [52]. Without detailed knowledge of the size and prevalence of all such substructure (particularly, the locally present substructure), it is difficult to place upper limits on the local dark matter density; however, an increase by greater than a factor of a few over the above estimates are unlikely [48]. On the other hand, one should in principle allow for the possibility that there may be some additional source of cold dark matter, such as axions, in which case the neutralino density would be less than $\rho_{0}$, though we do not consider this possibility here. Without a precise determination of $\rho_{0}$, it is difficult to place precise limits on the $\chi$-nucleon cross sections from direct detection experiments. However, the ratios of cross sections may be unambiguously determined, so we focus on these ratios in the following.

We take the fiducial values of the experimental quantities to be those obtained using the central values of the

TABLE II. Neutralino-nucleon scattering cross sections and ratios for benchmark models C, L, and M. Confidence intervals are given for confidence levels (C.L.) of $68.3 \%$ and $95.4 \%$, using the hadronic parameter uncertainties in Table I.

\begin{tabular}{|c|c|c|c|}
\hline Model & $\mathrm{C}$ & $\mathrm{L}$ & M \\
\hline$m_{1 / 2}(\mathrm{GeV})$ & 400 & 460 & 1840 \\
\hline$m_{0}(\mathrm{GeV})$ & 90 & 310 & 1400 \\
\hline $\tan \beta$ & 10 & 50 & 50 \\
\hline$A_{0}$ & 0 & 0 & 0 \\
\hline $\operatorname{sign}(\mu)$ & + & + & + \\
\hline$m_{\chi}(\mathrm{GeV})$ & 165 & 193 & 830 \\
\hline $\begin{array}{l}\sigma_{\chi \chi \mathrm{p}, \mathrm{SI}}(\mathrm{pb}) \\
68.3 \% \text { C.L. }\end{array}$ & $\begin{array}{c}2.85 \times 10^{-9} \\
(1.65-4.47) \times 10^{-9}\end{array}$ & $\begin{array}{c}2.36 \times 10^{-8} \\
(1.23-3.95) \times 10^{-8}\end{array}$ & $\begin{array}{c}1.28 \times 10^{-10} \\
(0.76-1.98) \times 10^{-10}\end{array}$ \\
\hline 95.4\% C.L. & $(0.81-6.46) \times 10^{-9}$ & $(0.49-5.98) \times 10^{-8}$ & $(0.39-2.83) \times 10^{-10}$ \\
\hline$\sigma_{\chi \mathrm{n}, \mathrm{SI}}(\mathrm{pb})$ & $2.93 \times 10^{-9}$ & $2.46 \times 10^{-8}$ & $1.32 \times 10^{-10}$ \\
\hline $68.3 \%$ C.L. & $(1.72-4.56) \times 10^{-9}$ & $(1.31-4.07) \times 10^{-8}$ & $(0.79-2.02) \times 10^{-10}$ \\
\hline 95.4\% C.L. & $(0.86-6.58) \times 10^{-9}$ & $(0.55-6.10) \times 10^{-8}$ & $(0.41-2.87) \times 10^{-10}$ \\
\hline$\sigma_{\chi \mathrm{p}, \mathrm{SD}}(\mathrm{pb})$ & $2.19 \times 10^{-6}$ & $1.82 \times 10^{-6}$ & $2.40 \times 10^{-8}$ \\
\hline 68.3\% C.L. & $(1.91-2.49) \times 10^{-6}$ & $(1.62-2.04) \times 10^{-6}$ & $(2.19-2.63) \times 10^{-8}$ \\
\hline 95.4\% C.L. & $(1.64-2.81) \times 10^{-6}$ & $(1.43-2.26) \times 10^{-6}$ & $(1.98-2.86) \times 10^{-8}$ \\
\hline$\sigma_{\chi \mathrm{n}, \mathrm{SD}}(\mathrm{pb})$ & $2.81 \times 10^{-6}$ & $2.10 \times 10^{-6}$ & $2.45 \times 10^{-8}$ \\
\hline 68.3\% C.L. & $(2.49-3.14) \times 10^{-6}$ & $(1.89-2.33) \times 10^{-6}$ & $(2.23-2.67) \times 10^{-8}$ \\
\hline 95.4\% C.L. & $(2.18-3.51) \times 10^{-6}$ & $(1.68-2.57) \times 10^{-6}$ & $(2.03-2.91) \times 10^{-8}$ \\
\hline$\sigma_{\chi \mathrm{n}, \mathrm{SI}} / \sigma_{\chi \mathrm{p}, \mathrm{SI}}$ & 1.029 & 1.042 & 1.026 \\
\hline $68.3 \%$ C.L. & $1.020-1.042$ & $1.028-1.06$ & $1.018-1.037$ \\
\hline 95.4\% C.L. & $1.015-1.066$ & $1.020-1.114$ & $1.013-1.056$ \\
\hline$\sigma_{\chi \mathrm{n}, \mathrm{SD}} / \sigma_{\chi \mathrm{p}, \mathrm{SD}}$ & 1.28 & 1.15 & 1.02 \\
\hline 68.3\% C.L. & $1.00-1.65$ & $0.93-1.44$ & $0.85-1.22$ \\
\hline 95.4\% C.L. & $0.78-2.14$ & $0.75-1.80$ & $0.71-1.460$ \\
\hline$\sigma_{\chi \mathrm{p}, \mathrm{SD}} / \sigma_{\chi \mathrm{p}, \mathrm{SI}}$ & 770 & 77 & 187 \\
\hline 68.3\% C.L. & $480-1350$ & $46-151$ & $121-319$ \\
\hline 95.4\% C.L. & $320-2730$ & $30-373$ & $83-616$ \\
\hline$\sigma_{\chi \mathrm{n}, \mathrm{SD}} / \sigma_{\chi \mathrm{n}, \mathrm{SI}}$ & 960 & 86 & 186 \\
\hline 68.3\% C.L. & $610-1660$ & $51-163$ & $120-313$ \\
\hline 95.4\% C.L. & $410-3370$ & $33-392$ & $83-601$ \\
\hline
\end{tabular}




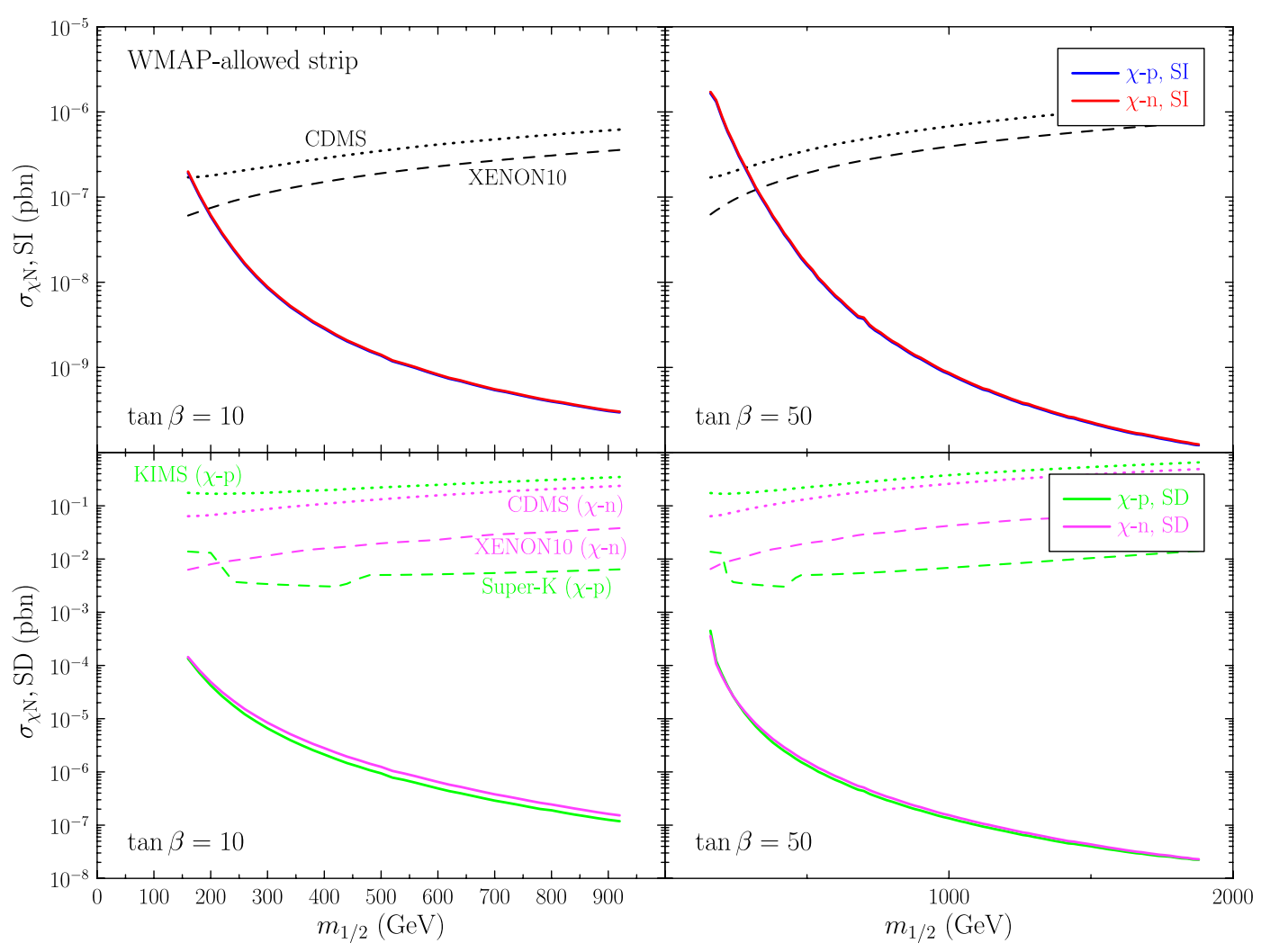

FIG. 1 (color online). The neutralino-nucleon scattering cross sections along the WMAP-allowed coannihilation strip for tan $\beta=10$ (left panels) and coannihilation/funnel strip for $\tan \beta=50$ (right panels) using the parameters in Table I. CMSSM predictions for the spin-independent (SI) cross sections are shown in the upper panels $\left(\sigma_{\chi \mathrm{p}, \mathrm{SI}}\right.$ in solid/blue, $\sigma_{\chi \mathrm{n}, \mathrm{SI}}$ in solid/red $)$, along with experimental constraints from CDMS (dotted/black line) and XENON10 (dashed/black line), where the constraints apply to either SI cross section under the reasonable MSSM assumption $\sigma_{\chi \mathrm{p}, \mathrm{SI}} \approx \sigma_{\chi \mathrm{n}, \mathrm{SI}}$. Note that $\sigma_{\chi \mathrm{p}, \mathrm{SI}}$ and $\sigma_{\chi \mathrm{n}, \mathrm{SI}}$ are nearly indistinguishable at the scale used for these plots. The lower panels show CMSSM predictions for the spin-dependent (SD) cross sections $\left(\sigma_{\chi \mathrm{p}, \mathrm{SD}}\right.$ in solid/green line, $\sigma_{\chi \mathrm{n}, \mathrm{SD}}$ in solid/magenta line). Experimental constraints for $\sigma_{\chi \mathrm{p}, \mathrm{SD}}$ are shown for KIMS (dotted/green line) and Super-Kamiokande (dashed/ green line), while constraints for $\sigma_{\chi \mathrm{n}, \mathrm{SD}}$ are given by CDMS (dotted/magenta line) and XENON10 (dashed/magenta line). Limits are based upon a local neutralino density of $0.3 \mathrm{GeV} / \mathrm{cm}^{3}$.

parameters in Table I. For illustration, cross sections and their ratios are given in Table II for a few benchmark models which are essentially the well-studied benchmark models C, L, and M [15]. All of the benchmark models lead to relic densities within the WMAP preferred range of $\Omega h^{2}=0.088-0.120$ [53], and satisfy most phenomenological constraints. ${ }^{3}$ Points $\mathrm{C}$ and $\mathrm{L}$ are points along the coannihilation strip (at low $\tan \beta=10$ and high $\tan \beta=$ 50 , respectively) where the masses of the neutralino and stau are nearly degenerate. Point $\mathrm{M}$ is in the funnel region where the mass of the neutralino is roughly half that of the Higgs pseudoscalar. Figure 1 shows the cross sections along the WMAP-allowed strips of the $\left(m_{1 / 2}, m_{0}\right)$ planes

\footnotetext{
${ }^{3}$ The exception being a possible failure to account for the discrepancy between the theoretical and experimental results for anomalous magnetic moment of the muon [54,55]. Points $\mathrm{C}$ and $\mathrm{L}$ are consistent with $(g-2)_{\mu}$, while the contribution from point $\mathrm{M}$ is too small.
}

for $\tan \beta=10$ and $50[1,16,56]$. For $\tan \beta=10$, this is the coannihilation strip and includes point $\mathrm{C}$, whereas for $\tan \beta=50$, the strip is formed by coannihilations at low $m_{1 / 2}$, turning into the rapid annihilation funnel at larger $m_{1 / 2}$. This strip includes points $\mathrm{L}$ and $\mathrm{M}$.

Current experimental upper limits on cross sections are also given in Fig. 1. All these limits assume a local neutralino density of $0.3 \mathrm{GeV} / \mathrm{cm}^{3}$, and should be rescaled by $\left(0.3 \mathrm{GeV} / \mathrm{cm}^{3}\right) / \rho_{0}$ for other values of $\rho_{0}$. We display the SI limits given by CDMS [30] and XENON10 [32] under the reasonable CMSSM assumption that $\sigma_{\chi \mathrm{p}, \mathrm{SI}} \approx \sigma_{\chi \mathrm{n}, \mathrm{SI}}$; relaxing that assumption would weaken the given limits by at most a factor of $\sim 5$. The same assumption cannot be made for the SD cross sections, and experiments typically constrain only one of $\sigma_{\chi \mathrm{p}, \mathrm{SD}}$ or $\sigma_{\chi \mathrm{n}, \mathrm{SD}}$, not both. The direct detection experiment KIMS [36] provides a limit for $\sigma_{\chi \mathrm{p}, \mathrm{SD}}$, while CDMS [31] and XENON10 [33] (taken from [57]) constrain $\sigma_{\chi \mathrm{n}, \mathrm{SD}}$. Since the Sun is primarily composed of hydrogen, the capture rate of neutralinos is 
particularly sensitive to $\sigma_{\chi \mathrm{p}, \mathrm{SD}}$; thus, indirect detection experiments are able to constrain this cross section. The large mass of the Sun and the large size of the detector allows Super-Kamiokande [38] to provide a significantly better limit on $\sigma_{\chi \mathrm{p}, \mathrm{SD}}$ than the direct detection experiments. It is apparent from Fig. 1 that experiments are beginning to probe the CMSSM parameter space through SI scattering, particularly at large $\tan \beta$, but are not yet sensitive enough to detect SD scattering.

From our analysis of the benchmark models and along the WMAP strip, several general features of the $\chi$-nucleon cross sections are apparent for any given CMSSM model. The SI cross sections for $\chi$-proton $\left(\sigma_{\chi \mathrm{p}, \mathrm{SI}}\right)$ and $\chi$-neutron $\left(\sigma_{\chi \mathrm{n}, \mathrm{SI}}\right)$ scattering are typically very close, within $\sim 5 \%$ of each other; close enough that they are virtually indistinguishable in Fig. 1. Likewise, the spin-dependent $\chi$-proton $\left(\sigma_{\chi \mathrm{p}, \mathrm{SD}}\right)$ and $\chi$-neutron $\left(\sigma_{\chi \mathrm{n}, \mathrm{SD}}\right)$ scattering cross sections are similar, differing by at most a factor of 2 or 3 . In general, the SD $\chi$-nucleon cross section is much larger than the SI one, by $\mathcal{O}\left(10^{2}-10^{3}\right)$ or more. However, we recall that the SI cross section for scattering off a nucleus, Eq. (9), contains a factor of the number of nucleons squared, whereas the SD cross section, Eq. (25), is proportional to the square of the spin, which does not grow with increasing nuclear mass. Consequently, heavy elements such as $\mathrm{Ge}$ and $\mathrm{Xe}$ are actually more sensitive to SI couplings than to SD couplings.

\section{A. Confidence intervals}

To determine the level of uncertainty in the cross sections and ratios obtained for a given model, we generate confidence intervals about the central values of the resulting cross section and ratio distributions by Monte Carlo sampling over the parameters in Table I, assuming independent Gaussian errors. ${ }^{4}$ Results are given in Table II for benchmark models $\mathrm{C}, \mathrm{L}$, and $\mathrm{M}$ at confidence levels (C.L.) of $68.3 \%$ and $95.4 \%$. These confidence intervals take into account only hadronic uncertainties, not any uncertainties arising within the supersymmetric models themselves. We recall, in particular, that the estimates for the $\alpha_{2 q}$ and $\alpha_{3 q}$ coefficients of the effective Lagrangian in Eq. (3) contain uncertainties that may only be reduced by calculating radiative corrections to the effective scattering operators, work that is beyond the scope of this paper. These confidence intervals take into account only the explicit dependence of $m_{\mathrm{t}}$ in Eqs. (4), (5), and (10), but not in MSSM terms such as $\theta_{\mathrm{t}}, m_{A}$, and $|\mu|$, which are calculated using the central value of $171.4 \mathrm{GeV}$. The confidence intervals

\footnotetext{
${ }^{4}$ The distributions for the cross sections generated by the Monte Carlo are not even approximately Gaussian or symmetric as is evidenced by the curvature seen in Fig. 2. Hence, confidence intervals are used instead of the averages and standard deviations of the generated distributions.
}

are not significantly affected by this incomplete treatment of the top-quark mass uncertainty, apart from a slight underestimate of the $\sigma_{\chi N, S D}$ interval widths; we shall address below the full MSSM dependence on the top-quark mass.

As indicated by the benchmark models, the hadronic uncertainties induce significant uncertainties in the cross sections. At the $68.3 \%$ C.L., the SI cross sections vary by $\sim 3$; at the $95.4 \%$ C.L., they vary by an order of magnitude. Such a variation is larger than might naïvely be expected given the much smaller relative uncertainties of the parameters listed in Table I, the largest of which is $m_{\mathrm{d}}$ at $5 \pm 2 \mathrm{MeV} .{ }^{5}$ Clearly, such a large uncertainty in the cross sections would make it difficult to narrow down CMSSM parameters on the basis of any single experimental signal.

Whilst the two SI cross sections can vary greatly, their ratio does not: in our Monte Carlo analysis, $\sigma_{\chi \mathrm{n}, \mathrm{SI}} / \sigma_{\chi \mathrm{p}, \mathrm{SI}}$ varies by only a few percent. It is worth noting, however, that in some models (e.g. model L), $\sigma_{\chi \mathrm{n}, \mathrm{SI}}$ may exceed $\sigma_{\chi \mathrm{p}, \mathrm{SI}}$ by more than $10 \%$ at the $95.4 \%$ C.L., a difference that may be of experimental interest. In addition, even at the $95.4 \%$ C.L., $\sigma_{\chi \mathrm{n}, \mathrm{SI}}$ is always larger than $\sigma_{\chi \mathrm{p}, \mathrm{SI}}$.

The variations in the two SD cross sections are not so large: $\sim 20 \%$ and $\sim 40 \%$ at the $68.3 \%$ and $95.4 \%$ C.L.'s, respectively. The ratio of the two cross sections, however, has a larger variation, typically by a factor of 2 or 3 , due to an anticorrelation in the variations of the two cross sections that we discuss below.

To demonstrate the contributions of individual parameters to the cross section uncertainties, we have determined $68.3 \%$ C.L. confidence intervals for variations in the parameters treated singly, with the remaining parameters fixed at their central values, as presented in Table III for benchmark model $\mathrm{C}$. The intervals are given as variations relative to the fiducial values. For several parameters, notably $\sigma_{0}, \Sigma_{\pi N}$, and $\Delta_{\mathrm{s}}^{(\mathrm{p})}$, the variations in the cross sections are nonlinear with respect to the parameters over the values of interest, resulting in confidence intervals that are not symmetric about the fiducial values. The variations due to the top mass here include the dependence of MSSM parameters such as $\theta_{\mathrm{t}}, A_{0}$, and $|\mu|$ on $m_{\mathrm{t}}$, which was neglected in the full confidence intervals of Table II.

It is clear that from Table III that the uncertainties in the quark masses and their ratios, apart from the top mass, make almost negligible contributions to the cross section uncertainties. The SI cross section uncertainties are dominated by contributions from $\sigma_{0}$ and $\Sigma_{\pi N}$, and the SD cross section uncertainties mainly arise from uncertainties in $\Delta_{\mathrm{s}}^{(\mathrm{p})}$. We examine these in the following sections.

\footnotetext{
${ }^{5}$ We show below that, while having the largest relative uncertainty, $m_{\mathrm{d}}$ makes a negligible contribution to the uncertainties in the cross sections.
} 
TABLE III. Relative uncertainties in $\sigma_{\chi \mathrm{p}, \mathrm{SI}}, \sigma_{\chi \mathrm{p}, \mathrm{SD}}, \sigma_{\chi \mathrm{n}, \mathrm{SI}} / \sigma_{\chi \mathrm{p}, \mathrm{SI}}$, and $\sigma_{\chi \mathrm{n}, \mathrm{SD}} / \sigma_{\chi \mathrm{p}, \mathrm{SD}}$ for Model $\mathrm{C}$ due to each of the parameters in Table I. The quoted uncertainties correspond to $68.3 \%$ C.L. confidence intervals relative to the fiducial values. Uncertainties in $\sigma_{\chi \mathrm{n}, \mathrm{SI}}$ and $\sigma_{\chi \mathrm{n}, \mathrm{SD}}$ (not shown) are comparable to those in $\sigma_{\chi \mathrm{p}, \mathrm{SI}}$ and $\sigma_{\chi \mathrm{p}, \mathrm{SD}}$, respectively. Because of the nonlinear dependence of the cross sections on some of the parameters over the ranges of interest, some confidence intervals are not symmetric about the fiducial values.

\begin{tabular}{lcccc}
\hline \hline & $\sigma_{\chi \mathrm{p}, \mathrm{SI}}(\mathrm{pb})$ & $\sigma_{\chi \mathrm{p}, \mathrm{SD}}(\mathrm{pb})$ & $\sigma_{\chi \mathrm{n}, \mathrm{SI}} / \sigma_{\chi \mathrm{p}, \mathrm{SI}}$ & $\sigma_{\chi \mathrm{n}, \mathrm{SD}} / \sigma_{\chi \mathrm{p}, \mathrm{SD}}$ \\
\hline fiducial value & $2.85 \times 10^{-9}$ & $2.19 \times 10^{-6}$ & 1.029 & 1.28 \\
$m_{\mathrm{u}} / m_{\mathrm{d}}$ & $\pm 3.5 \%$ & $\sim 0^{\mathrm{a}}$. & $\pm 0.08 \%$ & $\sim 0$. \\
$m_{\mathrm{d}}$ & $\sim 0$. & $\sim 0$. & $\sim 0$. & $\sim 0$. \\
$m_{\mathrm{S}} / m_{\mathrm{d}}$ & $\pm 5.2 \%$ & $\sim 0$. & $\pm 0.07 \%$ & $\sim 0$. \\
$m_{\mathrm{c}}$ & $\sim 0$. & $\sim 0$. & $\sim 0$. & $\sim 0$. \\
$m_{\mathrm{b}}$ & $\pm 0.1 \%$ & $\pm 0.04 \%$ & $\sim 0$. & $\pm 0.01 \%$ \\
$m_{\mathrm{t}}$ & $\pm 9 \%$ & $\pm 9 \%$ & $\pm 0.005 \%$ & $\pm 2 \%$ \\
$\sigma_{0}$ & $+34 \%$ & - & $-0.7 \%$ & - \\
$\Sigma_{\pi N}$ & $-27 \%$ & - & $-0.4 \%$ & - \\
$a_{3}^{(\mathrm{p})}$ & $-32 \%$ & $\pm 0.5 \%$ & - & $\pm 0.06 \%$ \\
$a_{8}^{(\mathrm{p})}$ & - & $\pm 2.2 \%$ & - & $\pm 4.2 \%$ \\
$\Delta_{\mathrm{s}}^{(\mathrm{p})}$ & - & $+14 \%$ & - & $+30 \%$ \\
\hline \hline
\end{tabular}

${ }^{a}$ Entries listed as $\sim 0$. have a relative variation less than $10^{-5}$ that of the fiducial value.

\section{B. Quark masses and mass ratios}

The most significant quark mass dependence of the $\chi$-nucleon scattering cross sections is that on the top-quark mass, which induces uncertainties of $\sim 10 \%$ in all four $\sigma_{\chi N}$. These uncertainties arise not from the appearance of $m_{\mathrm{t}}$ in Eqs. (4), (5), and (10) (hereafter referred to as the explicit $m_{\mathrm{t}}$ dependence), but in the calculations of CMSSM parameters, e.g., $m_{A}$ and $|\mu|$. However, variations in $m_{\mathrm{t}}$ rescale all four cross sections in the same manner, so that ratios of the cross sections are essentially independent of $m_{\mathrm{t}}$. The precision of the top mass measurement will continue to improve once the LHC begins taking data, with a 1 $\mathrm{GeV}$ uncertainty a possibility in as little as $1 \mathrm{yr}$ of lowluminosity running [58]. Thus, we can expect the $m_{\mathrm{t}}$-induced uncertainties in $\sigma_{\chi N}$ to fall from $\sim 10 \%$ to $\sim 5 \%$ or lower within a few years, well below uncertainties induced by other parameters.

The SI cross sections are sensitive to the ratios of the light quark masses $m_{\mathrm{u}} / m_{\mathrm{d}}$ and $m_{\mathrm{s}} / m_{\mathrm{d}}$, more than to the overall scale of the light quark masses, which is fixed by the relatively poorly constrained value of $m_{\mathrm{d}}$. In the CMSSM, $\theta_{f} \approx 0$ for the light quarks $(u, d, s)$ and the charm quark (c), so that $\eta$ in Eq. (2) is nearly diagonal. For diagonal $\eta, \alpha_{3 q}$ is proportional to $m_{q}$, and hence $\alpha_{3 q} / m_{q}$ in Eq. (10) is independent of the quark mass. Any dependence on the light quark masses must come through the $f_{T q}^{(\mathrm{p}, \mathrm{n})}$ terms. However, by Eqs. (19)-(21), those terms depend only on mass ratios. Since there is no dependence on $m_{\mathrm{d}}$ (for fixed mass ratios), its relatively large uncertainty does not translate into any significant uncertainties in the SI cross sections.
Whilst the mass ratios induce uncertainties of a few percent in the cross sections, the induced uncertainties in the ratio $\sigma_{\chi \mathrm{n}, \mathrm{SI}} / \sigma_{\chi \mathrm{p}, \mathrm{SI}}$ are only $\sim 0.1 \%$. This is a consequence of the fact that the predominant contribution to Eq. (10) comes from the strange-quark term $f_{T \mathrm{~s}}^{(\mathrm{p}, \mathrm{s})} \alpha_{3 \mathrm{~s}} / m_{\mathrm{s}}$, which is common to both $f_{\mathrm{p}}$ and $f_{\mathrm{n}}$. Thus, variations in the quark mass ratios induce, via Eq. (21), comparable (and correlated) variations in both $\sigma_{\chi \mathrm{p}, \mathrm{SI}}$ and $\sigma_{\chi \mathrm{n}, \mathrm{SI}}$, resulting in only small variations in the ratio of those two cross sections.

The $\eta$ matrices for the bottom and top quarks, unlike the lighter quarks, are not approximately diagonal, so that $\alpha_{3 q} / m_{q}$ does have a quark mass dependence. However, the precisions to which these quark masses are known lead to only small uncertainties $(<0.1 \%)$ in the SI cross sections (here, we refer only to the explicit $m_{\mathrm{t}}$ dependence in these terms, not to the MSSM parameter dependence discussed previously).

The only quark mass dependence of the SD cross sections in Eq. (27) comes from the light quark masses in $\alpha_{2 q}$ $(q=u, d, s)$. However, mass-dependent terms are suppressed by a factor of $\mathcal{O}\left(m_{q} / m_{W}\right)\left(\mathcal{O}\left(m_{q}^{2} / m_{W}^{2}\right)\right.$ for diagonal $\eta$ matrices) relative to mass-independent terms arising from the $X_{i}, Y_{i}, W_{i}$, and $V_{i}$ factors given by Eq. (6). Thus, the SD cross sections are nearly independent of the quark masses.

\section{Spin-independent parameters and cross sections}

The greatest impediment to an accurate determination of the SI cross sections for any given MSSM model comes 


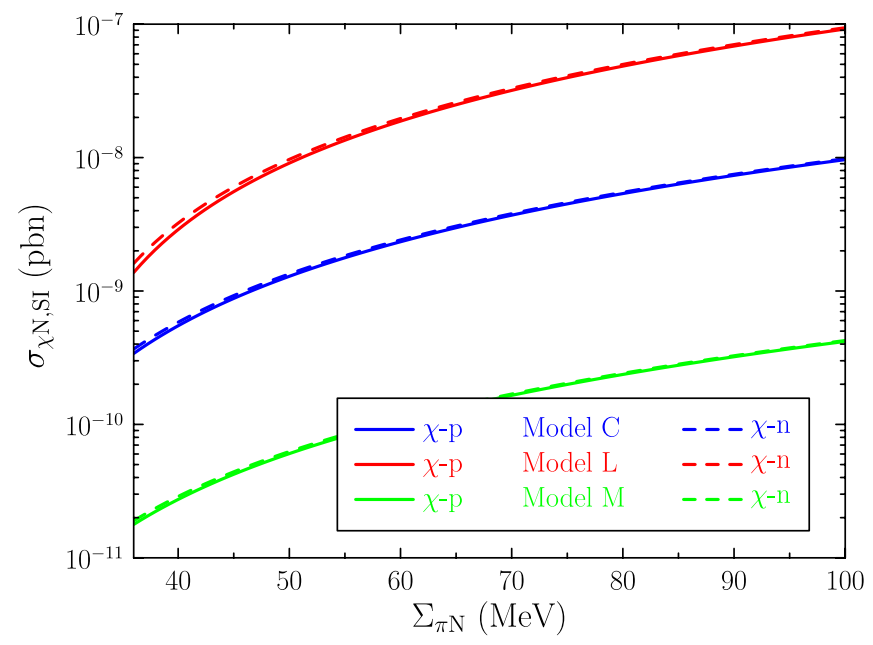

FIG. 2 (color online). The spin-independent neutralinonucleon scattering cross section as a function of $\Sigma_{\pi N}$ for benchmark models C, L, and M. Note that $\sigma_{\chi \mathrm{p}, \mathrm{SI}}$ and $\sigma_{\chi \mathrm{n}, \mathrm{SI}}$ are nearly indistinguishable at the scale used in this plot.

from the $\Sigma_{\pi N}$ and $\sigma_{0}$ parameters. As shown for benchmark model C in Table III, each of these two parameters induces uncertainties of $\sim 30 \%$ or more in $\sigma_{\chi \mathrm{p}, \mathrm{SI}}$ at the $68.3 \%$ C.L.; $\sigma_{\chi \mathrm{n}, \mathrm{SI}}($ not shown) has similar induced uncertainties. The large confidence intervals for the SI cross sections in Table II are almost entirely due to the uncertainties in these two parameters.

We focus the discussion here mainly on $\Sigma_{\pi N}$ rather than $\sigma_{0}$ as the $\Sigma_{\pi N}=64 \pm 8 \mathrm{MeV}$ result is significantly larger than previous estimates for that parameter. Indeed the range of estimates of the central value for $\Sigma_{\pi N}$ is far greater than the typically quoted uncertainty. In view of this, we also include below some results for lower values of $\Sigma_{\pi N}$.

In Fig. 2, we show the $\Sigma_{\pi_{N}}$ dependence of $\sigma_{\chi^{N, S I}}$ for the benchmark models, and Table IV gives the $\sigma_{\chi N, S I}$ values for those models for selected values of $\Sigma_{\pi N}$. All the other parameters are set at their fiducial values (Table I). From the minimal value for $\Sigma_{\pi N}\left(\sigma_{0}=36 \mathrm{MeV}\right)$ to the $2-\sigma$ upper bound $(80 \mathrm{MeV}), \sigma_{\chi N, S I}$ varies by more than a factor of 10 (as much as a factor of 35 for model L). At these benchmarks and in other models of interest, for larger values of $\Sigma_{\pi N}\left(\Sigma_{\pi N} \nsucc \sigma_{0}\right)$, the majority of the contribution to $f_{\mathrm{p}}$ in Eq. (10) comes from the strange-quark term, with $f_{T_{s}}^{(\mathrm{p})} \propto y \propto \Sigma_{\pi N}-\sigma_{0}$, so that $\sigma_{\chi \mathrm{p}, \mathrm{SI}} \sim\left(\Sigma_{\pi N}-\sigma_{0}\right)^{2}$. Thus, the SI cross sections are particularly sensitive not just to $\Sigma_{\pi N}$ and $\sigma_{0}$, but to their difference. For smaller values of $\Sigma_{\pi N}\left(\Sigma_{\pi N} \sim \sigma_{0}\right)$, the strange contribution no longer dominates, but a strong dependence of $\sigma_{\chi N, S I}$ on $\Sigma_{\pi N}$ does remain.

In Fig. 3, the SI cross sections are shown along the WMAP-allowed coannihilation strip for $\tan \beta=10$ and coannihilation/funnel strip for $\tan \beta=50$ for the $\Sigma_{\pi N}$
TABLE IV. Spin-independent neutralino-nucleon scattering cross sections in the benchmark models for several values of $\Sigma_{\pi N}$

\begin{tabular}{cccc}
\hline \hline Model & $\mathrm{C}$ & $\mathrm{L}$ & $\mathrm{M}$ \\
\hline$\Sigma_{\pi N}=36 \mathrm{MeV}:$ & & & \\
$\sigma_{\chi \mathrm{p}, \mathrm{SI}}(\mathrm{pb})$ & $3.40 \times 10^{-10}$ & $1.38 \times 10^{-9}$ & $1.78 \times 10^{-11}$ \\
$\sigma_{\chi \mathrm{n}, \mathrm{SI}}(\mathrm{pb})$ & $3.67 \times 10^{-10}$ & $1.61 \times 10^{-9}$ & $1.89 \times 10^{-11}$ \\
$\sigma_{\chi \mathrm{n}, \mathrm{SI}} / \sigma_{\chi \mathrm{p}, \mathrm{SI}}$ & 1.080 & 1.170 & 1.065 \\
$\Sigma_{\pi N}=45 \mathrm{MeV}:$ & & & \\
$\sigma_{\chi \mathrm{p}, \mathrm{SI}}(\mathrm{pb})$ & $8.80 \times 10^{-10}$ & $5.55 \times 10^{-9}$ & $4.23 \times 10^{-11}$ \\
$\sigma_{\chi \mathrm{n}, \mathrm{SI}}(\mathrm{pb})$ & $9.24 \times 10^{-10}$ & $6.02 \times 10^{-9}$ & $4.41 \times 10^{-11}$ \\
$\sigma_{\chi \mathrm{n}, \mathrm{SI}} / \sigma_{\chi \mathrm{p}, \mathrm{SI}}$ & 1.050 & 1.085 & 1.043 \\
$\Sigma_{\pi N}=56 \mathrm{MeV}:$ & & & \\
$\sigma_{\chi \mathrm{p}, \mathrm{SI}}(\mathrm{pb})$ & $1.88 \times 10^{-9}$ & $1.45 \times 10^{-8}$ & $8.64 \times 10^{-11}$ \\
$\sigma_{\chi \mathrm{n}, \mathrm{SI}}(\mathrm{pb})$ & $1.95 \times 10^{-9}$ & $1.52 \times 10^{-8}$ & $8.91 \times 10^{-11}$ \\
$\sigma_{\chi \mathrm{n}, \mathrm{SI}} / \sigma_{\chi \mathrm{p}, \mathrm{SI}}$ & 1.035 & 1.053 & 1.031 \\
$\Sigma_{\pi N}=64 \mathrm{MeV}:$ & & & \\
$\sigma_{\chi \mathrm{p}, \mathrm{SI}}(\mathrm{pb})$ & $2.85 \times 10^{-9}$ & $2.36 \times 10^{-8}$ & $1.28 \times 10^{-10}$ \\
$\sigma_{\chi \mathrm{n}, \mathrm{SI}}(\mathrm{pb})$ & $2.93 \times 10^{-9}$ & $2.46 \times 10^{-8}$ & $1.32 \times 10^{-10}$ \\
$\sigma_{\chi \mathrm{n}, \mathrm{SI}} / \sigma_{\chi \mathrm{p}, \mathrm{SI}}$ & 1.029 & 1.042 & 1.026 \\
$\Sigma_{\pi N}=72 \mathrm{MeV}:$ & & & \\
$\sigma_{\chi \mathrm{p}, \mathrm{SI}}(\mathrm{pb})$ & $4.01 \times 10^{-9}$ & $3.49 \times 10^{-8}$ & $1.78 \times 10^{-10}$ \\
$\sigma_{\chi \mathrm{n}, \mathrm{SI}}(\mathrm{pb})$ & $4.11 \times 10^{-9}$ & $3.61 \times 10^{-8}$ & $1.82 \times 10^{-10}$ \\
$\sigma_{\chi \mathrm{n}, \mathrm{SI}} / \sigma_{\chi \mathrm{p}, \mathrm{SI}}$ & 1.025 & 1.035 & 1.022 \\
$\Sigma_{\pi N}=84 \mathrm{MeV}:$ & & & \\
$\sigma_{\chi \mathrm{p}, \mathrm{SI}}(\mathrm{pb})$ & $6.13 \times 10^{-9}$ & $5.61 \times 10^{-8}$ & $2.69 \times 10^{-10}$ \\
$\sigma_{\chi \mathrm{n}, \mathrm{SI}}(\mathrm{pb})$ & $6.26 \times 10^{-9}$ & $5.76 \times 10^{-8}$ & $2.74 \times 10^{-10}$ \\
$\sigma_{\chi \mathrm{n}, \mathrm{SI}} / \sigma_{\chi \mathrm{p}, \mathrm{SI}}$ & 1.021 & 1.028 & 1.019 \\
\hline \hline & & & \\
\hline
\end{tabular}

reference values of $36 \mathrm{MeV}$ (no strange scalar contribution), $64 \mathrm{MeV}$ (central value), and $80 \mathrm{MeV}$ (2- $\sigma$ upper bound); CDMS and XENON10 limits are also given. As with the benchmark models, a factor of $\sim 10$ variation occurs in $\sigma_{\chi N \text {,SI }}$ over these $\Sigma_{\pi N}$ reference values for any given model along the WMAP strip.

Such large variations present difficulties in using any upper limit or possible future precision measurement of $\sigma_{\chi N, S I}$ from a direct detection signal to constrain the CMSSM parameters. The present CDMS and XENON10 upper limits have (almost) no impact on the WMAP strip for $\tan \beta=10$ (50), if one makes the very conservative assumption that $\sigma_{0}=36 \mathrm{MeV}(y=0)$. On the other hand, $m_{1 / 2} \sim 200 \mathrm{GeV}$ would be excluded for $\tan \beta=10$ if $\Sigma_{\pi N}=64$ or $80 \mathrm{MeV}$. This excluded region would extend to $m_{1 / 2} \sim 300 \mathrm{GeV}$ for $\tan \beta=50$ if $\Sigma_{\pi N}=64$ or $80 \mathrm{MeV}$. Thus, the experimental uncertainty in $\Sigma_{\pi N}$ is already impinging on the ability of the present CDMS and XENON10 results to constrain the CMSSM parameter space.

Looking to the future, a conjectural future measurement of $\sigma_{\chi \mathrm{p}, \mathrm{SI}}=4 \times 10^{-9} \mathrm{pb}$ would only constrain $m_{1 / 2}$ to the range $600 \mathrm{GeV}<m_{1 / 2}<925 \mathrm{GeV}$ if $\tan \beta=10$ and $1100 \mathrm{GeV}<m_{1 / 2}<1400 \mathrm{GeV}$ if $\tan \beta=50$, for the $1-\sigma$ $\Sigma_{\pi N}$ range of $64 \pm 8 \mathrm{MeV}$. If smaller values of $\Sigma_{\pi N}$ are 


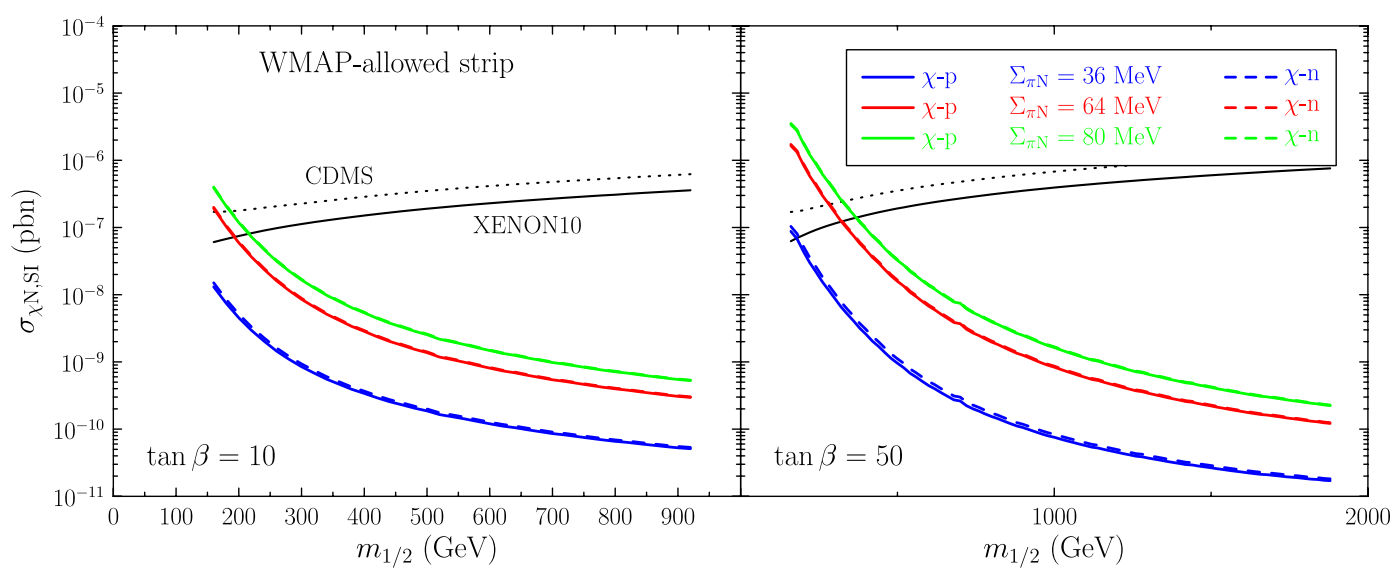

FIG. 3 (color online). The spin-independent neutralino-nucleon scattering cross section ratio along the WMAP-allowed strips for $\tan \beta=10$ (left panel) and $\tan \beta=50$ (right panel) for several values of $\Sigma_{\pi N}$. Experimental constraints from CDMS and XENON10 are also shown.

also considered, down to $\sigma_{0}=36 \mathrm{MeV}(y=0)$, these constraints would weaken to $350 \mathrm{GeV}<m_{1 / 2}<$ $925 \mathrm{GeV}$ and $550 \mathrm{GeV}<m_{1 / 2}<1400 \mathrm{GeV}$ for $\tan \beta=10$ and 50, respectively, ruling out only the smallest values of $m_{1 / 2}{ }^{6}$

As the SD cross sections are independent of $\Sigma_{\pi N}$, the SD/SI cross section ratio exhibits the same behavior with respect to $\Sigma_{\pi N}$ as the SI cross section does (albeit inverted), as shown in Fig. 4. The ratio is also shown along the corresponding WMAP strips in Fig. 5 for several values of $\Sigma_{\pi N}{ }^{7}$ For small $\Sigma_{\pi N}$, the ratio is large, almost $10^{4}$ for model $\mathrm{C}$ and 2000 for models $\mathrm{L}$ and $\mathrm{M}$ at $\Sigma_{\pi N}=36 \mathrm{MeV}$, suggesting that spin-sensitive experiments have a better chance of detecting neutralinos than spin-insensitive experiments. At the 2- $\sigma$ upper limit on $\Sigma_{\pi N}$, however, the ratio falls to 500 for model C, 50 for model $\mathrm{L}$, and 120 for model M. Recalling that the SI $\chi$-nuclear scattering cross section scales as the number of nucleons squared, SI scattering may actually dominate over SD scattering for heavier nuclei in the cases of these lower SD/SI cross section ratios.

The dependence of the SI $\chi$-n $/ \chi$-p cross section ratio on $\Sigma_{\pi N}$ is shown in Fig. 6 for the benchmark models, with ratios at selected $\Sigma_{\pi N}$ given in Table IV. The $\sigma_{\chi \mathrm{n}, \mathrm{SI}} / \sigma_{\chi \mathrm{p}, \mathrm{SI}}$ ratio is shown along the WMAP coannihilation strips in Fig. 7 for several values of $\Sigma_{\pi N}$. Whilst $\sigma_{\chi \mathrm{p}, \mathrm{SI}}$ and $\sigma_{\chi \mathrm{n}, \mathrm{SI}}$ each depend significantly on the value of $\Sigma_{\pi N}$, their ratio has only a mild $\Sigma_{\pi N}$ dependence. As noted earlier in this

\footnotetext{
${ }^{6}$ Moreover, as noted previously, however, detection signals only measure $\rho_{0} \sigma_{\chi N}$, so $\sigma_{\chi N}$ can only be determined from a signal to the precision that the local dark matter density is known.

${ }^{7}$ The nonmonotonic dependence on $m_{1 / 2}$ seen in the right panel of Fig. 5 for $\tan \beta=50$ is due to an enhancement of the Higgsino components, $Z_{\chi_{3}}$ and $Z_{\chi^{4}}$, at low $m_{1 / 2}$. As a result, the $Z$-exchange contribution to the spin-dependent cross section increases rapidly.
}

section, the strange-quark term dominates in Eq. (10) for $\Sigma_{\pi N}+\sigma_{0}$ and, since this term contributes identically to both $f_{\mathrm{p}}$ and $f_{\mathrm{n}}$ (neglecting nucleon mass differences), $\sigma_{\chi \mathrm{n}, \mathrm{SI}} / \sigma_{\chi \mathrm{p}, \mathrm{SI}} \approx 1$, independent of $\Sigma_{\pi N}$. For $\Sigma_{\pi N} \sim \sigma_{0}$, where the strange contribution is no longer significant, we generally find that $f_{T_{\mathrm{u}}}, f_{T_{\mathrm{d}}} \ll 1$ so that $f_{T G} \approx 1$ via Eq. (12) and the right (heavy-quark) summation term in Eq. (10) dominates over the left (light-quark) summation term. Since the heavy-quark terms are identical in $f_{\mathrm{p}}$ and $f_{\mathrm{n}}$, we again have $\sigma_{\chi \mathrm{n}, \mathrm{SI}} / \sigma_{\chi \mathrm{p}, \mathrm{SI}} \approx 1$.

The $f_{T_{\mathrm{u}}}^{(N)}$ and $f_{T_{\mathrm{d}}}^{(N)}$ terms are small but not entirely negligible, and the fact that $f_{T_{q}}^{(\mathrm{p})} \neq f_{T_{q}}^{(\mathrm{n})}$ for $q=u, d$ implies that $f_{\mathrm{p}} \neq f_{\mathrm{n}}$ in general. The result is a small shift in the SI $\chi$-n $/ \chi$-p cross section ratio away from unity. The shift becomes greater as $\Sigma_{\pi N} \rightarrow \sigma_{0}$, since the dominant strange-quark contribution disappears and allows the pro-

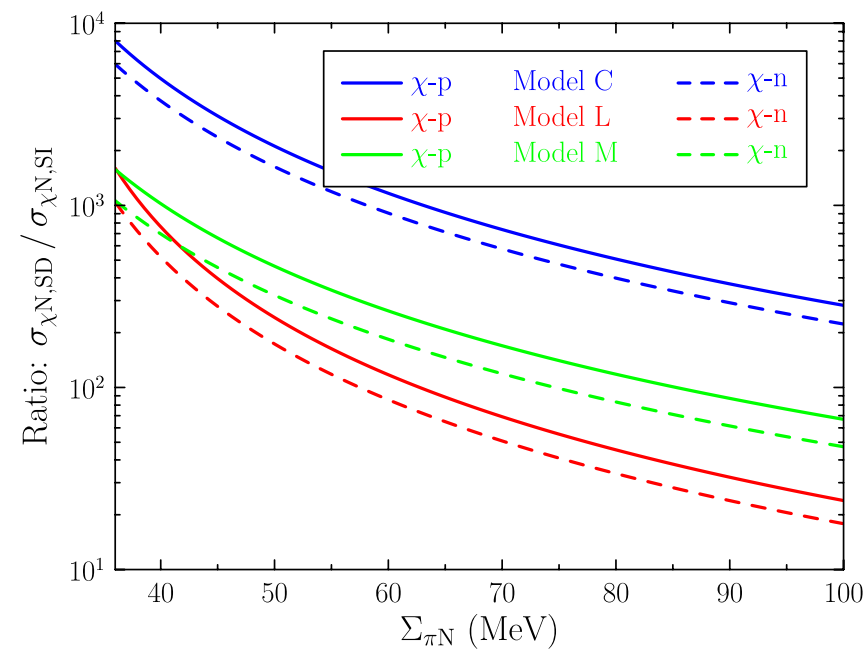

FIG. 4 (color online). The ratios of the spin-dependent and spin-independent neutralino-nucleon scattering cross sections as functions of $\Sigma_{\pi N}$ for the benchmark models C, L, and M. 


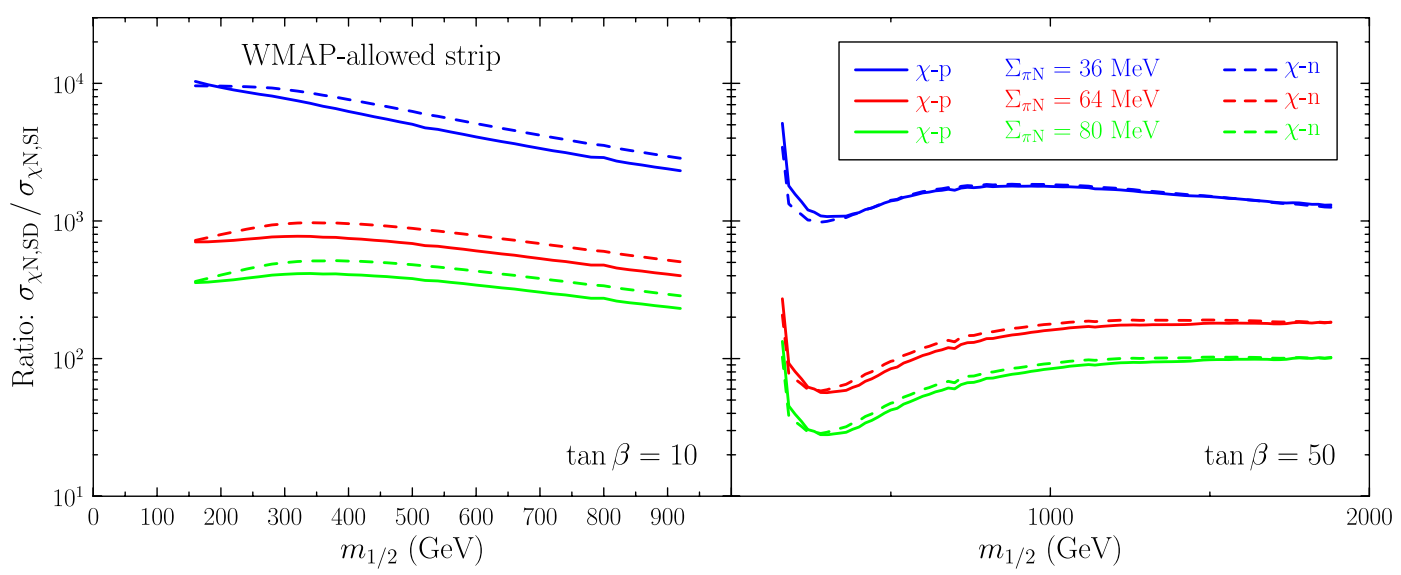

FIG. 5 (color online). The ratios of the spin-dependent and spin-independent neutralino-nucleon scattering cross sections along the WMAP-allowed strips for $\tan \beta=10$ (left panel) and $\tan \beta=50$ (right panel) for several values of $\Sigma_{\pi N}$.

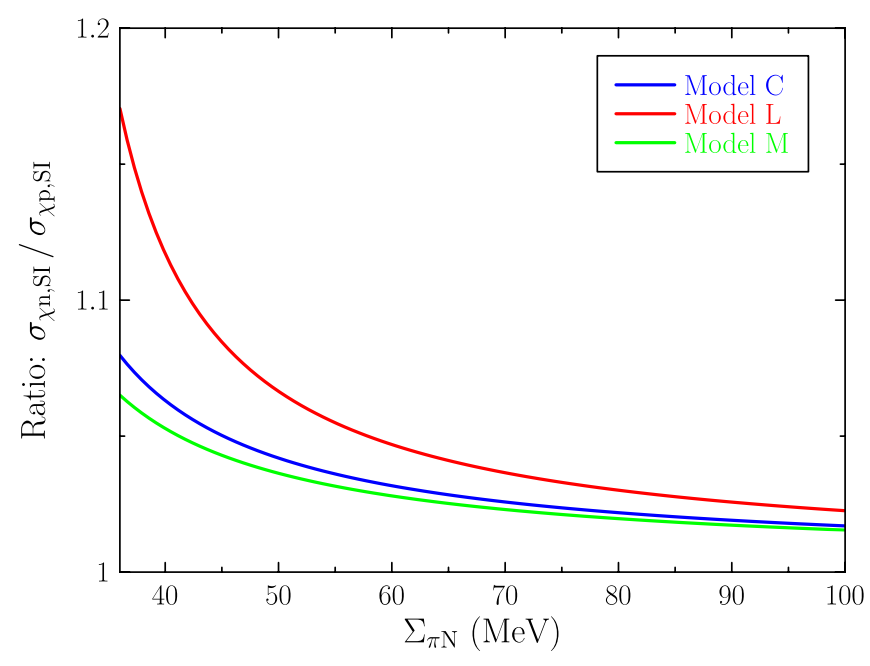

FIG. 6 (color online). The ratios of the spin-independent neutralino-neutron and neutralino-proton scattering cross sections as functions of $\Sigma_{\pi N}$ for benchmark models C, L, and M. ton/neutron asymmetry in the smaller up and down quark terms to become relevant. For the benchmark models in Fig. 6 and along the WMAP coannihilation strips in Fig. 7, $\sigma_{\chi \mathrm{n}, \mathrm{SI}}$ is $\sim 5 \%$ larger than $\sigma_{\chi \mathrm{p}, \mathrm{SI}}$ in the $2-\sigma \Sigma_{\pi N}$ range $64 \pm$ $16 \mathrm{MeV}$. For $\Sigma_{\pi N} \sim \sigma_{0}$, however, the difference can become as large as $15 \%$ to $20 \%$.

It can be shown that $\sigma_{\chi \mathrm{n}, \mathrm{SI}} / \sigma_{\chi \mathrm{p}, \mathrm{SI}}>1$ for $\left|\alpha_{3 \mathrm{~d}}\right|>\left|\alpha_{3 \mathrm{u}}\right|$. Since $\alpha_{3 \mathrm{u}} \propto 1 / B_{\mathrm{u}}=1 / \sin \beta$ and $\alpha_{3 \mathrm{~d}} \propto 1 / B_{\mathrm{d}}=1 / \cos \beta$, that condition is generally satisfied for the $\tan \beta \gg 1$ models examined here. Hence, the neutron always has a slightly larger SI cross section than the proton in these models.

When including all the uncertainties, as shown by the Monte Carlo results in Table II, a small but nonzero asymmetry in the proton and neutron SI cross sections is expected to occur at the $\sim$ few $\%$ level. Such an asymmetry may be observable, but a problem arises in the current generation of experiments. To extract the relative strength of the $f_{\mathrm{p}}$ and $f_{\mathrm{n}}$ couplings in Eq. (9), a signal must be seen in two detectors with different ratios of $Z$ and $A-Z$. However, to boost the scattering cross section and, there-

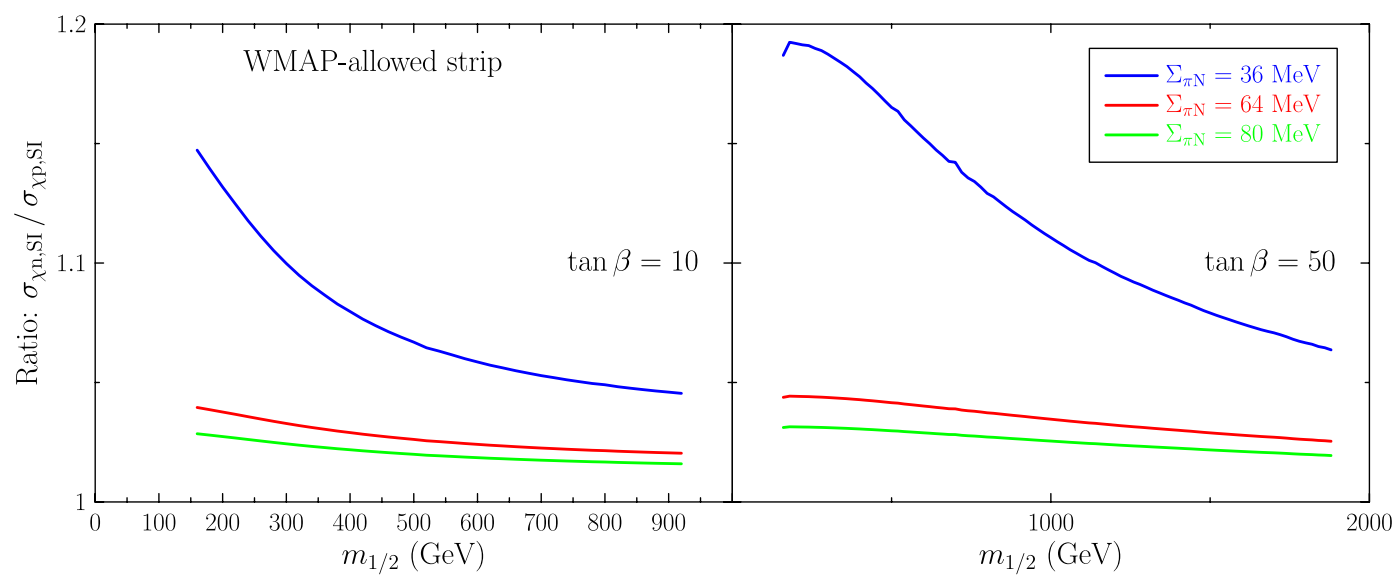

FIG. 7 (color online). The ratios of the spin-independent neutralino-neutron and neutralino-proton scattering cross sections along the WMAP-allowed strips for $\tan \beta=10$ (left panel) and $\tan \beta=50$ (right panel) for several values of $\Sigma_{\pi N}$. 
TABLE V. The spin-dependent neutralino-nucleon scattering cross sections in the benchmark models for several values of $\Delta_{\mathrm{s}}^{(\mathrm{p})}$.

\begin{tabular}{cccc}
\hline \hline Model & $\mathrm{C}$ & $\mathrm{L}$ & $\mathrm{M}$ \\
\hline$\Delta_{\mathrm{s}}^{(\mathrm{p})}=-0.15:$ & & & \\
$\sigma_{\chi \mathrm{p}, \mathrm{SD}}(\mathrm{pb})$ & $2.80 \times 10^{-6}$ & $2.25 \times 10^{-6}$ & $2.86 \times 10^{-8}$ \\
$\sigma_{\chi \mathrm{n}, \mathrm{SD}}(\mathrm{pb})$ & $2.19 \times 10^{-6}$ & $1.69 \times 10^{-6}$ & $2.03 \times 10^{-8}$ \\
$\sigma_{\chi \mathrm{n}, \mathrm{SD}} / \sigma_{\chi \mathrm{p}, \mathrm{SD}}$ & 0.78 & 0.75 & 0.71 \\
$\Delta_{\mathrm{s}}^{(\mathrm{p})}=-0.12:$ & & & \\
$\sigma_{\chi \mathrm{p}, \mathrm{SD}}(\mathrm{pb})$ & $2.48 \times 10^{-6}$ & $2.03 \times 10^{-6}$ & $2.63 \times 10^{-8}$ \\
$\sigma_{\chi \mathrm{n}, \mathrm{SD}}(\mathrm{pb})$ & $2.49 \times 10^{-6}$ & $1.89 \times 10^{-6}$ & $2.23 \times 10^{-8}$ \\
$\sigma_{\chi \mathrm{n}, \mathrm{SD}} / \sigma_{\chi \mathrm{p}, \mathrm{SD}}$ & 1.00 & 0.93 & 0.85 \\
$\Delta_{\mathrm{s}}^{(\mathrm{p})}=-0.09:$ & & & \\
$\sigma_{\chi \mathrm{p}, \mathrm{SD}}(\mathrm{pb})$ & $2.19 \times 10^{-6}$ & $1.82 \times 10^{-6}$ & $2.40 \times 10^{-8}$ \\
$\sigma_{\chi \mathrm{n}, \mathrm{SD}}(\mathrm{pb})$ & $2.81 \times 10^{-6}$ & $2.10 \times 10^{-6}$ & $2.45 \times 10^{-8}$ \\
$\sigma_{\chi \mathrm{n}, \mathrm{SD}} / \sigma_{\chi \mathrm{p}, \mathrm{SD}}$ & 1.28 & 1.15 & 1.02 \\
$\Delta_{\mathrm{s}}^{(\mathrm{p})}=-0.06:$ & & & \\
$\sigma_{\chi \mathrm{p}, \mathrm{SD}}(\mathrm{pb})$ & $1.91 \times 10^{-6}$ & $1.63 \times 10^{-6}$ & $2.19 \times 10^{-8}$ \\
$\sigma_{\chi \mathrm{n}, \mathrm{SD}}(\mathrm{pb})$ & $3.14 \times 10^{-6}$ & $2.33 \times 10^{-6}$ & $2.67 \times 10^{-8}$ \\
$\sigma_{\chi \mathrm{n}, \mathrm{SD}} / \sigma_{\chi \mathrm{p}, \mathrm{SD}}$ & 1.64 & 1.43 & 1.22 \\
$\Delta_{\mathrm{s}}^{(\mathrm{p})}=-0.03:$ & & & \\
$\sigma_{\chi \chi \mathrm{p}, \mathrm{SD}}(\mathrm{pb})$ & $1.65 \times 10^{-6}$ & $1.44 \times 10^{-6}$ & $1.99 \times 10^{-8}$ \\
$\sigma_{\chi \mathrm{n}, \mathrm{SD}}(\mathrm{pb})$ & $3.49 \times 10^{-6}$ & $2.57 \times 10^{-6}$ & $2.90 \times 10^{-8}$ \\
$\sigma_{\chi \mathrm{n}, \mathrm{SD}} / \sigma_{\chi \mathrm{p}, \mathrm{SD}}$ & 2.11 & 1.78 & 1.46 \\
$\Delta_{\mathrm{s}}^{(\mathrm{p})}=0.00:$ & & & \\
$\sigma_{\chi \mathrm{p}, \mathrm{SD}}(\mathrm{pb})$ & $1.41 \times 10^{-6}$ & $1.26 \times 10^{-6}$ & $1.80 \times 10^{-8}$ \\
$\sigma_{\chi \mathrm{n}, \mathrm{SD}}(\mathrm{pb})$ & $3.86 \times 10^{-6}$ & $2.81 \times 10^{-6}$ & $3.15 \times 10^{-8}$ \\
$\sigma_{\chi \mathrm{n}, \mathrm{SD}} / \sigma_{\chi \mathrm{p}, \mathrm{SD}}$ & 2.74 & 2.23 & 1.75 \\
\hline \hline & & & \\
\hline
\end{tabular}

fore, neutralino detection likelihood, many current experiments use heavy elements such as Ge and Xe that have similar ratios $A-Z \approx 1.4 Z$. It will be necessary to see a signal in experiments using lighter elements nearer to $A-Z \approx Z$ (i.e. $Z \lesssim 17$ ) in order to determine $\sigma_{\chi \mathrm{n}, \mathrm{SI}} / \sigma_{\chi \mathrm{p}, \mathrm{SI}}$. Such experiments are possible, but their SI sensitivities typically lag far behind those with heavier elements.

\section{Spin-dependent parameters and cross sections}

The determination of the SD cross sections for a given MSSM model depends on the three parameters specifying the spin content in a nucleon: $a_{3}^{(\mathrm{p})}, a_{8}^{(\mathrm{p})}$, and $\Delta_{\mathrm{s}}^{(\mathrm{p})}$. As demonstrated for benchmark model $\mathrm{C}$ in Table III, uncertainties in $a_{3}^{(\mathrm{p})}$ and $a_{8}^{(\mathrm{p})}$ induce only $<1 \%$ and $\sim$ few $\%$ uncertainties in $\sigma_{\chi N, \mathrm{SD}}$, respectively, at the 1- $\sigma$ level. Uncertainties in the strange spin contribution $\Delta_{\mathrm{s}}^{(\mathrm{p})}$, on the other hand, induce 10 to $15 \%$ uncertainties in $\sigma_{\chi^{N, S D}}$, and uncertainties in this parameter account for essentially all the width of the SD confidence intervals in Table II. Since uncertainties induced by $a_{3}^{(\mathrm{p})}$ and $a_{8}^{(\mathrm{p})}$ are negligible, we ignore these terms and focus on $\Delta_{\mathrm{s}}^{(\mathrm{p})}$.

We give in Table $\mathrm{V}$ the $\mathrm{SD}$ cross sections and their ratios for the benchmark models for several $\Delta_{\mathrm{s}}^{(\mathrm{p})}$ values ranging from -0.15 (the $2-\sigma$ lower bound) to 0.0 (no strange contribution to the nucleon spin). Over the 2- $\sigma$ range $-0.09 \pm 0.06$, both $\sigma_{\chi \mathrm{p}, \mathrm{SD}}$ and $\sigma_{\chi \mathrm{n}, \mathrm{SD}}$ vary by $\sim 40 \%$ in each of the three models, a significant variation but not so large as that induced in the SI cross sections by $\Sigma_{\pi N}$. However, unlike $\Sigma_{\pi N}$ and the SI cross sections, the SD proton and neutron cross sections are anti-correlated with $\Delta_{\mathrm{s}}^{(\mathrm{p})}$ : as the value of $\Delta_{\mathrm{s}}^{(\mathrm{p})}$ increases, $\sigma_{\chi \mathrm{p}, \mathrm{SD}}$ decreases while $\sigma_{\chi \mathrm{n}, \mathrm{SD}}$ increases.

Figures 8 and 9 show the SD cross sections and the SD/ SI cross section ratios along the WMAP coannihilation strips for several values of $\Delta_{\mathrm{s}}^{(\mathrm{p})}$. These figures again demonstrate the anticorrelation of the neutron/proton cross sections with $\Delta_{\mathrm{s}}^{(\mathrm{p})}$ as (for decreasing $\Delta_{\mathrm{s}}^{(\mathrm{p})}$ ) $\sigma_{\chi \mathrm{p}, \mathrm{SD}}$ and $\sigma_{\chi \mathrm{n}, \mathrm{SD}}$ approach each other and, for low enough $\Delta_{\mathrm{s}}^{\mathrm{p})}$, cross over ( $\sigma_{\chi \mathrm{p}, \mathrm{SD}}$ becomes the larger SD cross section).

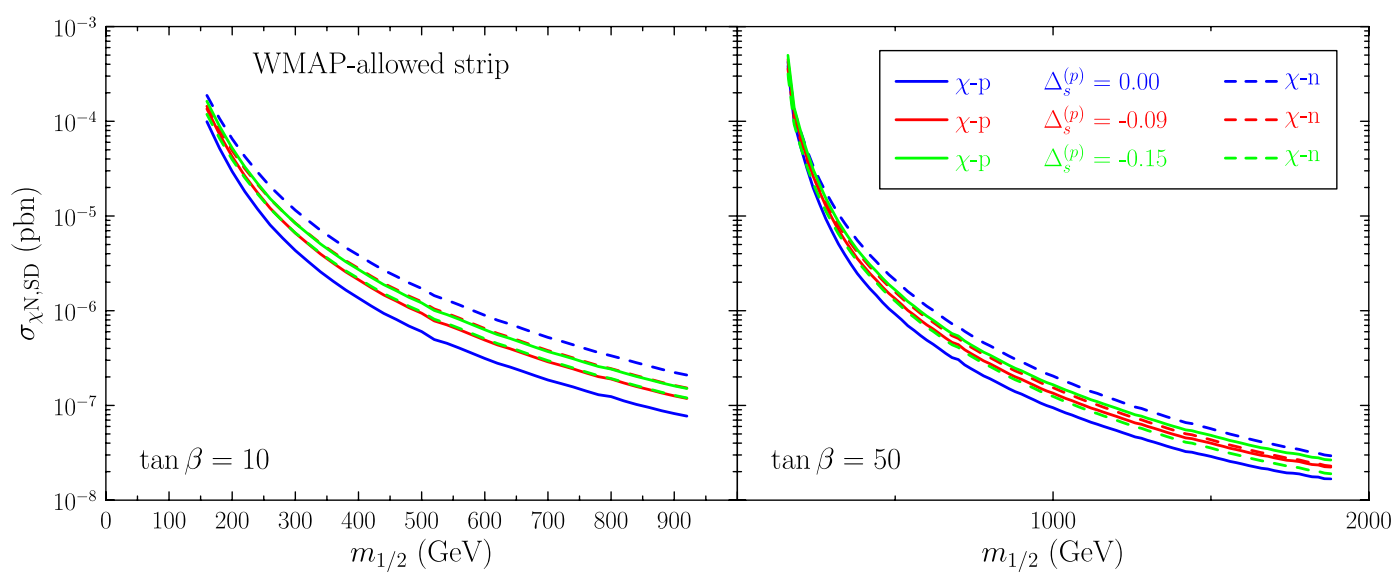

FIG. 8 (color online). The ratios of the spin-dependent neutralino-nucleon scattering cross sections along the WMAP-allowed strips for $\tan \beta=10$ (left panel) and $\tan \beta=50$ (right panel) for several values of $\Delta_{\mathrm{s}}^{(\mathrm{p})}$. 


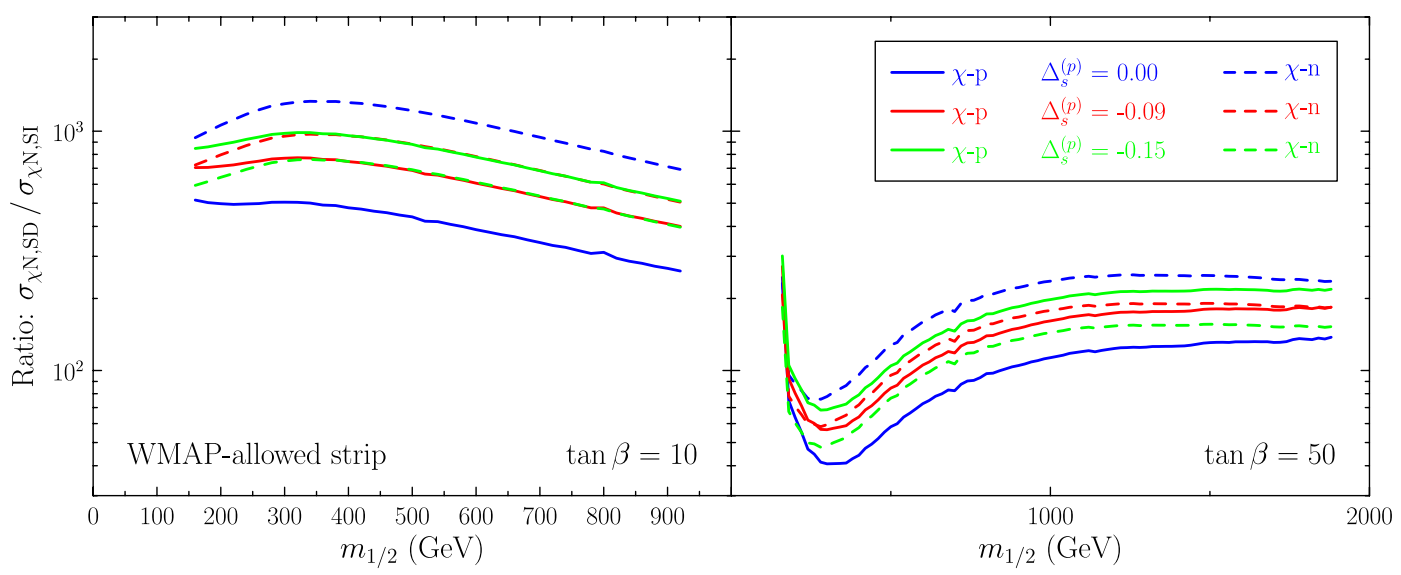

FIG. 9 (color online). The ratios of the spin-dependent and spin-independent neutralino-nucleon scattering cross sections along the WMAP-allowed strips for $\tan \beta=10$ (left panel) and $\tan \beta=50$ (right panel) for several values of $\Delta_{\mathrm{s}}^{(\mathrm{p})}$.

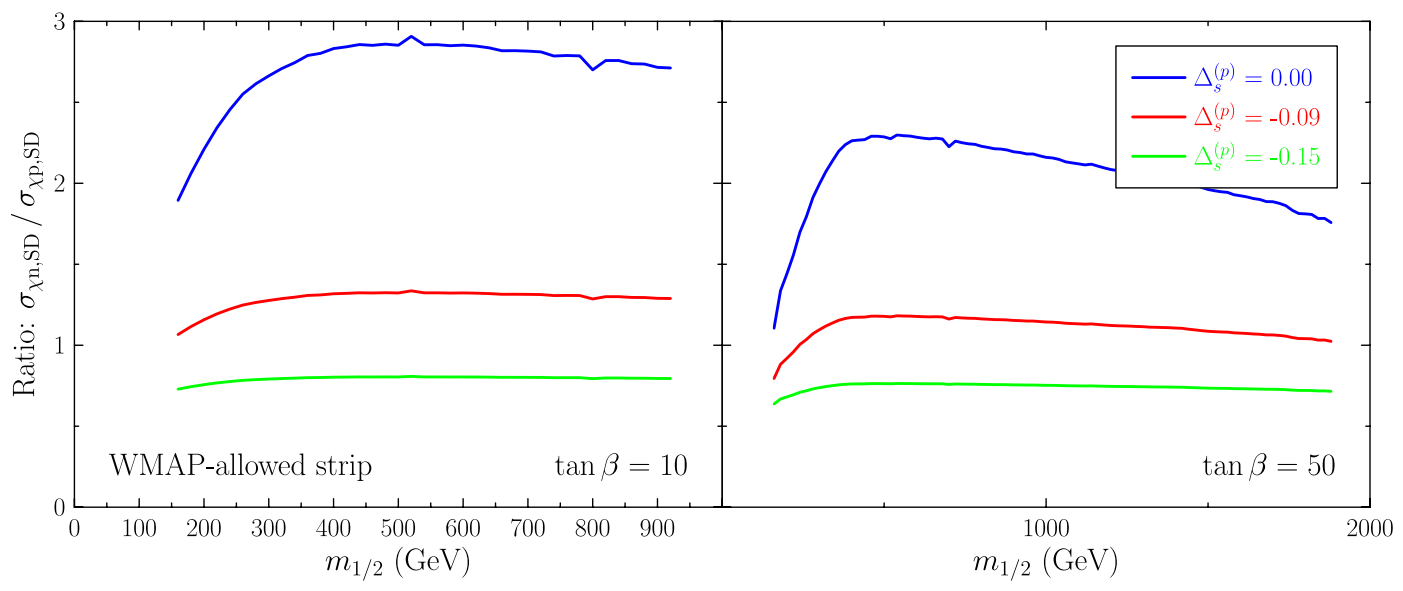

FIG. 10 (color online). The ratios of the spin-dependent neutralino-neutron and neutralino-proton scattering cross sections along the WMAP-allowed strips for $\tan \beta=10$ (left panel) and $\tan \beta=50$ (right panel) for several values of $\Delta_{\mathrm{s}}^{(\mathrm{p})}$.

Because of the anticorrelated behaviors of the SD cross sections, the $\mathrm{SD} \chi-\mathrm{n} / \chi-\mathrm{p}$ cross section ratio is particularly sensitive to the value of $\Delta_{\mathrm{s}}^{(\mathrm{p})}$. This ratio is shown along the WMAP coannihilation strips in Fig. 10, where it is apparent that the ratio varies by a factor of 2 to 3 at the $\sim 2-\sigma$ level for $\Delta_{\mathrm{s}}^{(\mathrm{p})}$. That factor of 2 to 3 in the ratio can also be seen in the confidence intervals at the $95.4 \%$ C.L., given in Table II.

The large uncertainty in $\sigma_{\chi \mathrm{n}, \mathrm{SD}} / \sigma_{\chi \mathrm{p}, \mathrm{SD}}$ induced by $\Delta_{\mathrm{s}}^{(\mathrm{p})}$ for a given model is unfortunate since this ratio may be one of the easiest to determine experimentally. This is because many elements have a spin-odd proton group $\left(\left\langle S_{\mathrm{p}}\right\rangle \neq 0\right)$ and a spin-even neutron group $\left(\left\langle S_{\mathrm{p}}\right\rangle \approx 0\right)$ or vice versa, in which case $\Lambda \propto a_{\mathrm{p}}$ or $\Lambda \propto a_{\mathrm{n}}$ in Eq. (25). By using an oddeven element in a detector, any signal could essentially be entirely attributed to $a_{\mathrm{p}}$ and therefore yield $\sigma_{\chi \mathrm{p}, \mathrm{SD}}$. Likewise, when using an even-odd element, any signal could essentially be entirely attributed to $a_{\mathrm{n}}$ and therefore yield $\sigma_{\chi \mathrm{n}, \mathrm{SD}}$. Even though the measurements would proba- bly actually be of $\rho_{0} \sigma_{\chi \mathrm{p}, \mathrm{SD}}$ and $\rho_{0} \sigma_{\chi \mathrm{n}, \mathrm{SD}}$ and not of $\sigma_{\chi \mathrm{p}, \mathrm{SD}}$ and $\sigma_{\chi \mathrm{n}, \mathrm{SD}}$ alone, the ratio $\sigma_{\chi \mathrm{n}, \mathrm{SD}} / \sigma_{\chi \mathrm{p}, \mathrm{SD}}$ is still an unambiguous and straightforward experimental measurement. The large uncertainties induced by $\Delta_{\mathrm{s}}^{(\mathrm{p})}$, however, make it difficult to use such a signal to constrain the CMSSM parameter space.

\section{SUMMARY AND DISCUSSION}

We have analyzed in this paper the principal hadronic uncertainties in the spin-independent (SI) and spindependent (SD) cross sections for supersymmetric relic scattering on protons and neutrons, using three benchmark points and two coannihilation strips as illustrations. We have found that the principal hadronic uncertainty in the SD cross sections is due to our lack of knowledge of the $\pi$-nucleon $\sigma$ term. In comparison, uncertainties in quark masses and their ratios are much less important. In the case of the SD cross sections, the dominant uncertainty is due to our ignorance of the strange-quark contribution to the 
nucleon spin, though this uncertainty is relatively less important than that induced in the SI cross sections by the $\pi$-nucleon $\sigma$ term.

This uncertainty in the $\pi$-nucleon $\sigma$ term clouds very significantly the interpretation of searches for (and eventually measurements of) dark matter scattering on nuclei, preventing precise answers to the key questions: How do present unsuccessful searches constrain the supersymmetric model parameter space? How accurately could a possible future measurement be used to refine the model parameters? This hadronic uncertainty is much larger than that generates by uncertainties in supersymmetric model calculations of the effective LSP-quark interactions, and also much larger than the astrophysical uncertainty in the local cold dark matter density.

One of the great hopes in supersymmetric phenomenology is that one will eventually be able to use measurements at accelerators such as the LHC and/or a linear $e^{+} e^{-}$ collider to calculate the relic LSP density in the Universe, and the rates for dark matter scattering. The hadronic uncertainty in the latter that is induced by our ignorance of the $\pi$-nucleon $\sigma$ term limits severely the prospects for completing the second part of this programme. Specifically, this uncertainty is much larger than the uncertainty in calculating the relic LSP density that could be expected from LHC measurements in at least one benchmark model.

We therefore plead for an experimental campaign to determine better the $\pi$-nucleon $\sigma$ term. This quantity is certainly interesting and important in its own right and as a measure of the importance of strange quarks in the nucleon. However, as argued in this paper, it is potentially also a key ingredient in the effort to understand one of the most important aspects of possible new physics beyond the standard model.

\section{ACKNOWLEDGMENTS}

The work of KAO was supported in part by DOE Grant No. DE-FG02-94ER-40823. C. S. acknowledges the support of the William I. Fine Theoretical Physics Institute at the University of Minnesota and thanks L. Duong for useful conversations.
[1] See, for example: K. A. Olive, arXiv:0709.3303.

[2] C. Savage, P. Gondolo, and K. Freese, Phys. Rev. D 70, 123513 (2004).

[3] G. Bertone, D. G. Cerdeno, J. I. Collar, and B. C. Odom, Phys. Rev. Lett. 99, 151301 (2007).

[4] H. Leutwyler, Phys. Lett. B 378, 313 (1996).

[5] W. M. Yao et al. (Particle Data Group), J. Phys. G 33, 1 (2006); 2007 partial update for the 2008 edition available on the PDG WWW (http://pdg.lbl.gov/).

[6] A.P. Heinson (CDF and D0 Collaboration), in Ninth Conference on the Intersections of Particle and Nuclear Physics, edited by T. M. Liss, AIP Conf. Proc. No. 870 (AIP, New York, 2006), p. 223.

[7] B. Borasoy and U. G. Meissner, Ann. Phys. (N.Y.) 254, 192 (1997).

[8] M. M. Pavan, I. I. Strakovsky, R. L. Workman, and R. A. Arndt, PiN Newslett. 16, 110 (2002).

[9] J. R. Ellis, K. A. Olive, Y. Santoso, and V. C. Spanos, Phys. Rev. D 71, 095007 (2005).

[10] Y. Goto et al. (Asymmetry Analysis Collaboration), Phys. Rev. D 62, 034017 (2000).

[11] E. Leader, A. V. Sidorov, and D. B. Stamenov, Phys. Rev. D 67, 074017 (2003).

[12] M. Alekseev et al. (COMPASS Collaboration), arXiv:0707.4077.

[13] J. R. Ellis, J. S. Hagelin, D. V. Nanopoulos, K. A. Olive, and M. Srednicki, Nucl. Phys. B238, 453 (1984); see also H. Goldberg, Phys. Rev. Lett. 50, 1419 (1983).

[14] M. Drees and M. M. Nojiri, Phys. Rev. D 47, 376 (1993); H. Baer and M. Brhlik, Phys. Rev. D 53, 597 (1996); 57, 567 (1998); J. R. Ellis, T. Falk, G. Ganis, K. A. Olive, and
M. Schmitt, Phys. Rev. D 58, 095002 (1998); V. D. Barger and C. Kao, Phys. Rev. D 57, 3131 (1998); J. R. Ellis, T. Falk, G. Ganis, K. A. Olive, and M. Srednicki, Phys. Lett. B 510, 236 (2001); L. Roszkowski, R. Ruiz de Austri, and T. Nihei, J. High Energy Phys. 08 (2001) 024; A. B. Lahanas and V.C. Spanos, Eur. Phys. J. C 23, 185 (2002); A. Djouadi, M. Drees, and J. L. Kneur, J. High Energy Phys. 08 (2001) 055; U. Chattopadhyay, A. Corsetti, and P. Nath, Phys. Rev. D 66, 035003 (2002); J. R. Ellis, K. A. Olive, and Y. Santoso, New J. Phys. 4, 32 (2002); H. Baer, C. Balazs, A. Belyaev, J. K. Mizukoshi, X. Tata, and Y. Wang, J. High Energy Phys. 07 (2002) 050; R. Arnowitt and B. Dutta, arXiv:hep-ph/0211417.

[15] M. Battaglia et al., Eur. Phys. J. C 22, 535 (2001); M. Battaglia, A. De Roeck, J.R. Ellis, F. Gianotti, K. A. Olive, and L. Pape, Eur. Phys. J. C 33, 273 (2004); A. De Roeck, J. R. Ellis, F. Gianotti, F. Moortgat, K. A. Olive, and L. Pape, Eur. Phys. J. C 49, 1041 (2007).

[16] J. R. Ellis, K. A. Olive, Y. Santoso, and V. C. Spanos, Phys. Lett. B 565, 176 (2003); H. Baer and C. Balazs, J. Cosmol. Astropart. Phys. 05 (2003) 006; A. B. Lahanas and D. V. Nanopoulos, Phys. Lett. B 568, 55 (2003); U. Chattopadhyay, A. Corsetti, and P. Nath, Phys. Rev. D 68, 035005 (2003); C. Munoz, Int. J. Mod. Phys. A 19, 3093 (2004).

[17] H. P. Nilles, Phys. Rep. 110, 1 (1984).

[18] H. E. Haber and G. L. Kane, Phys. Rep. 117, 75 (1985).

[19] T. Falk, A. Ferstl, and K. A. Olive, Phys. Rev. D 59, 055009 (1999); 60, 119904(E) (1999).

[20] T. Falk, A. Ferstl, and K. A. Olive, Astropart. Phys. 13, 301 (2000). 
[21] P. F. Smith and J. D. Lewin, Phys. Rep. 187, 203 (1990); J. D. Lewin and P. F. Smith, Astropart. Phys. 6, 87 (1996).

[22] M. A. Shifman, A. I. Vainshtein, and V. I. Zakharov, Phys. Lett. 78B, 443 (1978).

[23] A. I. Vainshtein, V.I. Zakharov, and M. A. Shifman, Usp. Fiz. Nauk 131, 537 (1980) [Sov. Phys. Usp. 23, 429 (1980)].

[24] H. Y. Cheng, Phys. Lett. B 219, 347 (1989).

[25] J. R. Ellis, A. Ferstl, and K. A. Olive, Phys. Lett. B 481, 304 (2000); Phys. Rev. D 63, 065016 (2001); Phys. Lett. B 532, 318 (2002).

[26] A. Bottino, F. Donato, N. Fornengo, and S. Scopel, Astropart. Phys. 13, 215 (2000); 18, 205 (2002).

[27] J. Gasser, H. Leutwyler, and M.E. Sainio, Phys. Lett. B 253, 252 (1991).

[28] M. Knecht, PiN Newslett. 15, 108 (1999).

[29] M.E. Sainio, PiN Newslett. 16, 138 (2002).

[30] D. S. Akerib et al. (CDMS Collaboration), Phys. Rev. Lett. 96, 011302 (2006).

[31] D. S. Akerib et al. (CDMS Collaboration), Phys. Rev. D 73, 011102 (2006).

[32] J. Angle et al. (XENON Collaboration), Phys. Rev. Lett. 100, 021303 (2008); see also: http://xenon.astro.columbia.edu/.

[33] U. Oberlack et al. (XENON Collaboration), Report No. EPS-HEP2007, 2007.

[34] G. J. Alner et al., Astropart. Phys. 28, 287 (2007).

[35] G. J. Alner et al. (ZEPLIN-II Collaboration), Phys. Lett. B 653, 161 (2007).

[36] H. S. Lee et al., Phys. Rev. Lett. 99, 091301 (2007).

[37] M. W. Goodman and E. Witten, Phys. Rev. D 31, 3059 (1985).

[38] S. Desai et al. (Super-Kamiokande Collaboration), Phys. Rev. D 70, 083523 (2004); 70, 109901(E) (2004).

[39] A. Silvestri (IceCube Collaboration), Mod. Phys. Lett. A 22, 1769 (2007).

[40] J. Silk, K. A. Olive, and M. Srednicki, Phys. Rev. Lett. 55, 257 (1985); M. Srednicki, K. A. Olive, and J. Silk, Nucl. Phys. B279, 804 (1987); J. S. Hagelin, K. W. Ng, and K. A. Olive, Phys. Lett. B 180, 375 (1986); K. W. Ng, K. A. Olive, and M. Srednicki, Phys. Lett. B 188, 138 (1987); A. Bottino, V. de Alfaro, N. Fornengo, G. Mignola, and M. Pignone, Phys. Lett. B 265, 57 (1991); K. Freese and M. Kamionkowski, Phys. Rev. D 55, 1771 (1997); L. Bergstrom, J. Edsjo, and P. Gondolo, Phys. Rev. D 58, 103519 (1998); V. D. Barger, F. Halzen, D. Hooper, and C. Kao, Phys. Rev. D 65, 075022 (2002); V. Barger, W. Y. Keung, G. Shaughnessy, and A. Tregre, Phys. Rev. D 76, 095008 (2007).

[41] K. Freese, Phys. Lett. 167B, 295 (1986); L. M. Krauss,
M. Srednicki, and F. Wilczek, Phys. Rev. D 33, 2079 (1986).

[42] J. Silk and M. Srednicki, Phys. Rev. Lett. 53, 624 (1984); J. R. Ellis, R. A. Flores, K. Freese, S. Ritz, D. Seckel, and J. Silk, Phys. Lett. B 214, 403 (1988); F. W. Stecker, S. Rudaz, and T. F. Walsh, Phys. Rev. Lett. 55, 2622 (1985); A. Bottino, C. Favero, N. Fornengo, and G. Mignola, Astropart. Phys. 3, 77 (1995).

[43] V. Berezinsky, A. Bottino, and G. Mignola, Phys. Lett. B 325, 136 (1994); P. Gondolo and J. Silk, Phys. Rev. Lett. 83, 1719 (1999); G. Bertone, G. Sigl, and J. Silk, Mon. Not. R. Astron. Soc. 337, 98 (2002).

[44] J. Binney and S. Tremaine, Galactic Dynamics (Princeton University Press, Princeton, NJ, 1987) p. 226.

[45] G. Jungman, M. Kamionkowski, and K. Griest, Phys. Rep. 267, 195 (1996).

[46] E. I. Gates, G. Gyuk, and M. S. Turner, Astrophys. J. 449, L123 (1995).

[47] M. Kamionkowski and A. Kinkhabwala, Phys. Rev. D 57, 3256 (1998).

[48] M. Kamionkowski and S.M. Koushiappas, arXiv:0801.3269.

[49] A. A. Klypin, A. V. Kravtsov, O. Valenzuela, and F. Prada, Astrophys. J. 522, 82 (1999).

[50] B. Moore, S. Ghigna, F. Governato, G. Lake, T. Quinn, J. Stadel, and P. Tozzi, Astrophys. J. 524, L19 (1999).

[51] B. Yanny et al. (SDSS Collaboration), Astrophys. J. 588, 824 (2003); 605, 575(E) (2004); H. J. Newberg et al. (SDSS Collaboration), Astrophys. J. 596, L191 (2003); S. R. Majewski, M. F. Skrutskie, M. D. Weinberg, and J. C. Ostheimer, Astrophys. J. 599, 1082 (2003).

[52] K. Freese, P. Gondolo, H. J. Newberg, and M. Lewis, Phys. Rev. Lett. 92, 111301 (2004); K. Freese, P. Gondolo, and H. J. Newberg, Phys. Rev. D 71, 043516 (2005).

[53] D. N. Spergel et al. (WMAP Collaboration), Astrophys. J. Suppl. Ser. 170, 377 (2007).

[54] G. W. Bennett et al. (Muon Collaboration), Phys. Rev. Lett. 92, 161802 (2004); Phys. Rev. D 73, 072003 (2006).

[55] M. Davier, Nucl. Phys. B, Proc. Suppl. 169, 288 (2007); F. Jegerlehner, Acta Phys. Pol. B 38, 3021 (2007); J.P. Miller, E. de Rafael, and B.L. Roberts, Rep. Prog. Phys. 70, 795 (2007); S. Eidelman, ICHEP06, Moscow, 2006, see: http://ichep06.jinr.ru/reports/333_6s1_9p30_ Eidelman.pdf.

[56] J. R. Ellis, K. A. Olive, and P. Sandick, J. High Energy Phys. 06 (2007) 079.

[57] R. Gaitskill, V. Mandic, and J. Filippini, Dark Matter Limit Plot Generator, see: http://dmtools.berkeley.edu/ limitplots/.

[58] I. Borjanovic et al., Eur. Phys. J. C 39S2, 63 (2005). 